\title{
Conformal Scalar Fields and Chiral Splitting on Super Riemann Surfaces *
}

\author{
Eric D'Hoker ${ }^{1}$ and D.H. Phong ${ }^{2}$ \\ ${ }^{1}$ Department of Physics, University of California, Los Angeles, CA 90024, USA \\ ${ }^{2}$ Department of Mathematics, Columbia University, New York, NY 10027, USA
}

\begin{abstract}
We provide a complete description of correlation functions of scalar superfields on a super Riemann surface, taking into account zero modes and non-trivial topology. They are built out of chirally split correlation functions, or conformal blocks at fixed internal momenta. We formulate effective rules which determine these completely in terms of geometric invariants of the super Riemann surface. The chirally split correlation functions have non-trivial monodromy and produce single-valued amplitudes only upon integration over loop momenta. Our discussion covers the even spin structure as well as the odd spin structure case which had been the source of many difficulties in the past. Super analogues of Green's functions, holomorphic spinors, and prime forms emerge which should pave the way to function theory on super Riemann surfaces. In superstring theories, chirally split amplitudes for scalar superfields are crucial in enforcing the GSO projection required for consistency. However one really knew how to carry this out only in the operator formalism to oneloop order. Our results provide a way of enforcing the GSO projection to any loop.
\end{abstract}

\section{Introduction}

Closed fermionic strings are built out of independent left and right movers on the world-sheet. In the Ramond-Neveu-Schwarz formulation, the string coordinates are the world-sheet scalars $x^{\mu}$ and Majorana spinors $\psi_{-}^{\mu}, \psi_{+}^{\mu}$, and although both are space-time vectors, the theory actually carries space-time fermions [1]. A supersymmetric spectrum is obtained after performing the projection of GliozziScherk-Olive (GSO), which retains only the even G-parity states in the NeveuSchwarz sector, and the positive (space-time) chirality states in the Ramond sector [2]. On world-sheets of non-trivial topology, spinors such as $\psi_{-}^{\mu}, \psi_{+}^{\mu}$ require a spin structure - which specifies the sign ambiguity as the spinor is transported around a

\footnotetext{
* Work supported in part by the National Science Foundation under grants PHY-80-19754, PHY-86-13201 and DMS-87-04209
} 
homology generator. In functional quantization, the GSO projection is realized in all channels of a superstring diagram by summing over all spin structures independently for $\psi_{-}^{\mu}$ and $\psi_{+}^{\mu}[3,4]$. This summation is natural from a geometrical point of view since no spin structure on the surface is preferred. It is required for the unitarity of the theory; in particular states of odd G-parity or negative (spacetime) chirality fermions are never produced in pairs [5]. The independent sum over left and right world-sheet chiralities results in a spectrum that is a tensor product of left and right states with independent space-time supersymmetries: the so-called Type II theories, with their $\mathrm{N}=2$ low-energy supergravity limits [6]. In the heterotic string, only one world-sheet chiral half of the superstring is retained and assembled with half of the bosonic string, so that only one space-time supersymmetry survives $[3,7]$. Thus it is crucial for the very construction of the Type II and heterotic strings to be able to separate left from right movers and endow them with independent spin structures.

In practice there are many obstructions to realizing this prescription:

(a) The fields $x^{\mu}$ and $\psi^{\mu}$ are non-split in nature on surfaces with euclidian signature. This is clear for the $x^{\mu}$ field which is real, but there is a problem as well with the spinors $\psi_{-}^{\mu}, \psi_{+}^{\mu}$. Since on a surface with euclidian signature there are no Majorana-Weyl spinors, opposite chirality components $\psi_{+}^{\mu}$ and $\psi_{-}^{\mu}$ of a spinor are complex conjugates of one another and must carry the same spin structure. In particular chirally symmetric determinants can be regularized preserving reparametrization invariance, but determinants for chiral fields which should be their square roots will have anomalies.

(b) The RNS action given in (3.4-3.11) below and in general vertex operators must be invariant under local supersymmetry, and this forces the appearance of terms which explicitly couple opposite chiralities [8]. For example in the case of the action, this gives rise to the quartic coupling $\chi_{\bar{z}}^{+} \chi_{z}^{-} \psi_{+}^{\mu} \psi_{-}^{\mu}$ which prevents any naive splitting prescription.

(c) A final source of serious difficulties is the correlation functions of the matter fields $x^{\mu}$ and $\psi_{-}^{\mu}, \psi_{+}^{\mu}$. In fact the correlation function $\left\langle x^{\mu}(z) x^{\mu}(w)\right\rangle$ cannot be split into holomorphic and anti-holomorphic components due to the zero mode of the scalar Laplacian, and on world sheets of non-trivial topology, also to the presence of holomorphic one-forms. For odd spin structures, the $\psi_{-}^{\mu}, \psi_{+}^{\mu}$ fields have zero modes - holomorphic $1 / 2$-forms - which have to be projected out of the propagators $\left\langle\psi_{+}^{\mu}(z) \psi_{+}^{\mu}(w)\right\rangle$, breaking their meromorphicity.

All these phenomena seem to spoil the independence of left and right degrees of freedom on the surface. It should be noted that they arise only from the matter fields, and not from the additional ghost fields that are needed for the gauge-fixing of the superstring. From the outset, the ghost contributions are chirally split. From this point of view the difficulties we face in constructing superstrings stem from the very simplest superconformal field theory, namely that of scalar superfields.

A number of known results partially remedy some of the difficulties outlined above. The first is that the Cauchy-Riemann operators $\bar{\partial}_{n}$ acting on forms of weight $n$ depend holomorphically on moduli parameters. Thus there is an intimate 
relation between e.g. left movers on the surface - holomorphic fields in Euclidian signature - and holomorphicity on moduli space. In particular Belavin and Knizhnik [9] have shown that appropriately normalized determinants of Laplace operators $\Delta_{n}^{-}=\bar{\partial}_{n}^{\dagger} \bar{\partial}_{n}$ are the modulus squared of holomorphic functions on moduli space, up to a local "holomorphic" anomaly caused by insistence on reparametrization invariance:

$$
\delta_{\mu} \delta_{\bar{\mu}} \ln N_{n}=c_{n} \int d^{2} z \sqrt{g}\left(\nabla_{z} \mu \nabla_{\bar{z}} \bar{\mu}+R \mu \bar{\mu}\right) .
$$

Here $N_{n}$ denotes the determinant ratio

$$
N_{n}=\frac{\operatorname{det}^{\prime} \Delta_{n}^{-}}{\operatorname{det}\left\langle\phi_{a}^{n} \mid \phi_{b}^{n}\right\rangle \operatorname{det}\left\langle\phi_{a}^{1-n} \mid \phi_{b}^{1-n}\right\rangle} .
$$

$c_{n}$ is the same coefficient as that of the conformal anomaly

$$
c_{n}=6 n^{2}-6 n+1 \text {. }
$$

$\phi_{a}^{n}$ are the zero modes of $\bar{\partial}_{n}$, and $\mu=\mu_{\tilde{z}}^{z}$ is a Beltrami differential measuring the deformation of complex structures. This implies that the holomorphic anomalies for combined matter and ghost fields cancel for the bosonic string and the superstring, but only as far as determinants are concerned. For the bosonic string the partition function at each loop level can be written solely in terms of determinants

$$
Z_{B}=\int \phi_{1}^{2} \wedge \ldots \wedge \bar{\phi}_{3 h-3}^{2} N_{2} N_{0}^{-13}(\operatorname{det} \operatorname{Im} \Omega)^{-13} .
$$

Thus the Belavin-Knizhnik theorem suffices in this case to split $Z_{B}$ into a holomorphic and an anti-holomorphic factor

$$
Z_{B}=\int(\operatorname{det} \operatorname{Im} \Omega)^{-13} F \wedge \bar{F},
$$

where $F$ is a modular form of weight 13 . Applied to the superstring, the theorem insures that the product

$$
N_{0}^{-5} N_{1 / 2}^{5} N_{2} N_{3 / 2}^{-1},
$$

which constitutes part of any gauge-fixed amplitude is also the modulus squared of a holomorphic function $\mathscr{F}(m)$ on moduli space. The function $\mathscr{F}$ depends on spin structures (since $N_{1 / 2}$ and $N_{3 / 2}$ do) and is uniquely determined up to a constant phase. This means that we can extract chiral determinant products and circumvent the first difficulty presented by the non-chiral nature of the matter fields discussed in (a) above. In contrast with the bosonic string, however, even the Type II superstring partition function $Z_{I I}$ alone involves (besides a natural factor $(\operatorname{det} \operatorname{Im} \Omega)^{-5}$ which is the analogue of the factor $(\operatorname{det} \operatorname{Im} \Omega)^{-13}$ appearing in $Z_{B}$ ) correlation functions of the matter fields, so that we still have to resolve the difficulties of (b) and (c).

A second result is that the superstring action can be recast in terms of analytic supergeometry, so that correlation functions resulting from terms in the action can be handled by superholomorphic splitting. Supermoduli space is a complex supermanifold with moduli space as its body, and it carries a canonical complex structure induced from the complex structure of the individual super Riemann surfaces. The super-derivatives $\mathscr{D}_{-}^{n}$ depend holomorphically on supermoduli 
parameters, and we have the following super analogue of the holomorphic anomaly [10]:

$$
\delta_{H} \delta_{\bar{H}} \ln \frac{s \operatorname{det}^{\prime} \mathscr{D}_{+} \mathscr{D}_{-}^{n}}{s \operatorname{det}\left\langle\Phi_{a}^{n} \mid \Phi_{b}^{n}\right\rangle s \operatorname{det}\left\langle\Phi_{a}^{-n+1 / 2} \mid \Phi_{b}^{-n+1 / 2}\right\rangle}=\hat{c}_{n} \int \mathscr{L} .
$$

Here

$$
\hat{c}_{n}=4 n-1
$$

$H=H_{-}{ }^{z}$ is a super Beltrami differential, and $\mathscr{L}$ is a local super-reparametrization invariant function linear in $H$ and $\bar{H}$ and independent of $n$. Again the coefficient of the superholomorphic anomaly is the same as that of the super-Weyl anomaly given in [11-13], and the superholomorphic anomalies of matter and superghosts will cancel in the critical dimension $d=10$. Since the partition function can be expressed entirely in terms of super-determinants in the superfield formalism, this result exhibits the partition function as the modulus squared of a holomorphic function on supermoduli space, up to a factor of $(\operatorname{det} \operatorname{Im} \hat{\Omega})^{-5}$, where $\hat{\Omega}$ is the super period matrix. Only the case of even spin structures is needed here, since odd spin structures always admit a Dirac zero mode which kills off their contributions to the partition function. Thus the chiral mixing terms in the action still allow superholomorphic splitting. This may seem surprising, and is only possible because the supercomplex structure of supermoduli space itself mixes chiralities of the gravitino field $\chi_{\bar{z}}^{+}$to second order. More recent proofs of superholomorphic splitting for the partition function have since appeared in [14-16]. Although this result eliminates the difficulties of (a), (b), (c) that arise from the action, it provides no information on how to split chiralities when vertex operators are inserted. Furthermore it is still not clear how to make connection with the component formalism and modular forms.

The first goal of this paper is to show that all apparent obstructions to chirally splitting superstring amplitudes can be resolved completely upon fixing the internal loop momenta, and in the case of odd spin structures also the fermion zero mode. In [13] we have already presented the basic ingredients of this remarkable property of chiral splitting, and we have also argued that it is equivalent to the property of holomorphic splitting on supermoduli space discussed above. However the treatment of [13] was restricted to the case of exponential insertions only and to even spin structures, except for the torus where the odd spin structure case was also treated. Here we shall show that all amplitudes with external bosonic states are the modulus squared of functions holomorphic in supermoduli parameters and polarization tensors, meromorphic in the vertex insertion points, provided the internal loop momenta, and for odd spin structure the Dirac zero mode are fixed. This allows chiral splitting and GSO projection, which are required for the consistency of the superstring.

The second goal is to obtain a complete description of the correlation functions and conformal blocks in the conformal theory of scalar superfields. This description is succinctly summarized by a set of simple and explicit effective rules for evaluating the conformal blocks in both component and superfield formalisms. The rules incorporate all effects due to the non-trivial topology of the worldsheet. In general correlation functions in a conformal field theory are built out of conformal blocks. These are solutions to differential equations on the universal 
moduli curve resulting from Ward identities [17]. Conformal blocks will have monodromy as insertion points are moved around non-trivial cycles. This monodromy is of crucial importance, since it provides constraints on possible combinations of conformal blocks which can be formed to produce single-valued correlation functions. Although operator product expansions dictate the conformal weight, analyticity, and pole structure of conformal blocks, they provide no clue as to the monodromy. This deeper information can usually be obtained only with methods adapted to each conformal field theory, such as Feigin-Fuchs contours for the minimal models $[17,18]$ and bosonization for $c=1$ Gaussian and orbifold models [19]. Our effective rules will provide an explicit expression for the monodromy in the case of scalar superfields.

As a byproduct of splitting, we shall arrive at natural candidates for super abelian differentials, super period matrices, and super prime forms, in terms of which the chiral amplitudes can be recast. These are all analogues of fundamental concepts in the theory of Riemann surfaces, and should play a similar role in the study of superconformal field theories on super Riemann surfaces. The appearance of these global objects indeed indicates that the passage from local data in conformal field theory to global expressions is in general highly non-trivial.

It may be worthwhile to give at this point some qualitative indications as to why it is necessary to fix internal loop momenta and, for odd spin structure,the Dirac zero mode. In the operator language, a scalar field $x^{\mu}$ satisfying the equations of motion can be expanded as

$$
x^{\mu}(z, \bar{z})=x_{0}^{\mu}+p^{\mu} \ln |z|^{2}+\sum_{n=1}^{\infty} \alpha_{n}^{\mu} z^{n}+\sum_{n=1}^{\infty} \bar{\alpha}_{n}^{\mu} \bar{z}^{n} .
$$

The oscillator modes $\alpha_{n}^{\mu}$ and $\bar{\alpha}_{n}^{\mu}$ are naturally complex conjugates of one another and each can be considered a chiral half of $x^{\mu}$, and the collective coordinate $x_{0}^{\mu}$ effectively never enters any amplitude due to translation invariance. The momenta $p^{\mu}$ on the other hand do not admit a natural splitting and are at the origin of the non-split nature of the $x^{\mu}$ propagator. Fixing all momenta however will allow us to split $x^{\mu}$ and its correlation functions. A similar situation arises for the Dirac zero mode in the Ramond sector:

$$
\psi_{+}^{\mu}=\psi_{0,+}^{\mu}+\sum_{n=1}^{\infty} d_{n}^{\mu} z^{n} .
$$

Although $\psi_{0,+}^{\mu}$ has definite chirality, it must be correctly normalized and this again introduces non-holomorphic dependence. In fact working with fixed internal loop momenta (to be integrated over ultimately) is familiar from the dual model, where the closed string propagator is usually written as

$$
\frac{\delta\left(L_{0}-\bar{L}_{0}\right)}{L_{0}+\bar{L}_{0}-2}=\int d^{2} q q^{p^{2}+M^{2}-1} \bar{q}^{p^{2}+\bar{M}^{2}-1},
$$

so that holomorphically split expressions appear at fixed moduli $(q)$, but also at fixed momenta $\left(p^{\mu}\right)$. Similar ideas have been encountered in orbifold and toroidal compactifications, and in recent work of Verlinde and Verlinde [20].

As stressed in [13] the notions which arise out of a correct prescription for chiral splitting are also the ones needed for a consistent formulation of the 
superstring measure in covariant perturbation theory. We shall however discuss these last issues elsewhere.

\section{Scalar Fields on a Riemann Surface}

We shall need some standard facts about scalar fields on a Riemann surface $M$. We shall go over them in some detail, since one of our objectives is to find their super analogues on super Riemann surfaces. Let $d s^{2}=2 g_{z \bar{z}} d z d \bar{z}$ be a metric on $M$. The lagrangian for a scalar field $x$ is $\partial_{z} x \partial_{\bar{z}} x / 4 \pi$. The partition function is given by

$$
Z_{x}=\left(\frac{\operatorname{det}^{\prime} \Delta}{\int d^{2} z \sqrt{g}}\right)^{-1 / 2} \text {. }
$$

In view of the Belavin-Knizhnik theorem (1.1) we can cancel the holomorphic anomaly by introducing the Liouville action

$$
S\left(g_{z \bar{z}}\right)=\frac{1}{48 \pi} \int d^{2} z \partial_{z} \ln g_{z \bar{z}} \partial_{\bar{z}} \ln g_{z \bar{z}},
$$

and the inverse partition function $Z_{\Delta}$ of a chiral scalar field by

$$
\frac{\operatorname{det}^{\prime} \Delta}{\operatorname{det}\left\langle\omega_{I} \mid \omega_{J}\right\rangle \int d^{2} z \sqrt{g}}=\left|Z_{\Delta}\right|^{4} e^{-2 S\left(g_{z \bar{z}}\right)},
$$

where $\omega_{I}, I=1, \ldots, h$ is a basis of holomorphic 1 -forms.

It is convenient to choose this basis in the following way. Recall that the first homology group for a surface with $h$ holes can be generated by $2 h$ cycles $A_{I}, B_{J}, I$, $J=1, \ldots, h$, with the canonical intersection relations

$$
\#\left(A_{I}, B_{J}\right)=\delta_{I J}, \quad \#\left(A_{I}, A_{J}\right)=\#\left(B_{I}, B_{J}\right)=0 \text {. }
$$

We can now single out a basis $\omega_{I}$ by the duality requirement

$$
\oint_{A_{I}} \omega_{J}=\delta_{I J}
$$

The period matrix $\Omega_{I J}$ can then be defined by

$$
\oint_{B_{I}} \omega_{J}=\Omega_{I J} \text {. }
$$

The matrix $\Omega_{I J}$ is symmetric, and the matrix of inner products of holomorphic 1 -forms in (2.2) takes the simple form

$$
\left\langle\omega_{I} \mid \omega_{J}\right\rangle=2 i \operatorname{Im} \Omega_{I J}
$$

Under a deformation of metric the period matrix changes by

$$
\delta \Omega_{I J}=-\frac{i}{2} \int d^{2} z g^{z \bar{z}} \omega_{I} \omega_{J} \delta g_{\bar{z} \bar{z}}
$$

which confirms in particular that it is invariant under Weyl scalings and small reparametrizations. With this construction for the torus the period matrix reduces 
to a single complex parameter $\tau$, and the chiral partition function $Z_{\Delta}$ is just the Dedekind eta function $\eta(\tau)$.

Correlation functions of conformal fields can be written in terms of the theta function and the prime form. Recall that after fixing a homology basis, the $2^{2 h}$ spin structures can be indexed by their half-integer theta characteristics $\delta=\left(\delta_{I}^{\prime}, \delta_{I}^{\prime \prime}\right)$, $I=1, \ldots, h$. The parity of a spin structure is given by $4 \delta^{\prime} \delta^{\prime \prime}$, and is the same as the parity of its number of zero modes. Generically an even spin structure will have no zero mode, while an odd one will have exactly one. In this latter case it can even be written down explicitly. In fact let the theta function with characteristics be defined as (see e.g. [21, 22])

$\vartheta[\delta]\left(z_{I}, \Omega_{I J}\right)=\sum_{n_{I} \in Z^{h}} \exp \left(\pi i\left(n_{I}+\delta_{I}^{\prime}\right) \Omega_{I J}\left(n_{J}+\delta_{J}^{\prime}\right)+2 \pi i\left(n_{I}+\delta_{I}^{\prime}\right)\left(z_{I}+\delta_{I}^{\prime \prime}\right)\right)$.

If $\delta$ is odd, a holomorphic spinor $h_{\delta}$ with spin structure $\delta$ is obtained by setting

$$
h_{\delta}(z)=\left(\sum_{I=1}^{h} \partial_{I} \vartheta[\delta](0, \Omega) \omega_{I}(z)\right)^{1 / 2} \text {. }
$$

Next we construct the prime form:

$$
E(z, w)=\frac{\vartheta[\delta]\left(\int_{w}^{z} \omega_{I}, \Omega\right)}{h_{\delta}(z) h_{\delta}(w)},
$$

which is actually independent of the choice of the odd characteristic $\delta$. The prime form is a holomorphic form of type $(-1 / 2,0)$ in each of the variables $z$ and $w$, and should be viewed as defined on the universal covering of the surface $M$. Alternatively we can choose fixed representatives $A_{I}, B_{I}$ of the basis of homology classes along which the Riemann surface can be cut open into a polygonal domain $M_{\text {cut }}$ (see Fig. 1). Restricted to $M_{\text {cut }}$ the prime form is single-valued, but has monodromy. In other words its boundary values along the two edges corresponding to the same cut may not agree. If we denote by $z+A_{K}$ and $z+B_{K}$ points that are identified with $z$ after parallel transport along the cycles $A_{K}$ and $B_{K}$ respectively, then the monodromy of the prime form can be described as follows

$$
\begin{aligned}
& E\left(z+A_{K}, w\right)=E(z, w), \\
& E\left(z+B_{K}, w\right)=E(z, w) \exp \left(-\pi i \Omega_{K K}+2 \pi i \int_{z}^{w} \omega_{K}\right) .
\end{aligned}
$$

This implies immediately that

$$
\begin{aligned}
& \oint_{A_{I}} d z \partial_{z} \partial_{w} \ln E(z, w)=0, \\
& \oint_{B_{I}} d z \partial_{z} \partial_{w} \ln E(z, w)=2 \pi i \omega_{I}(w) .
\end{aligned}
$$

We note that

$$
\partial_{z} \partial_{w} \ln E(z, w)
$$




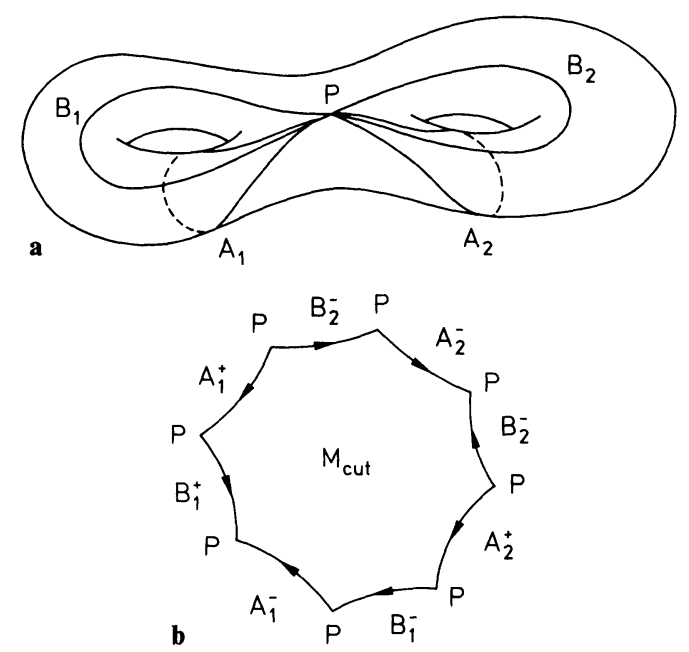

Fig. 1a, b. A basis of homology cycles. b The Riemann surface $M$ cut along the curves $A_{I}, B_{I}$

is just the abelian differential of the second kind with a double pole at $w$, and (2.11) is its usual normalization. Similarly

$$
\partial_{z} \ln \frac{E(z, w)}{E(z, P)}
$$

is a well-defined meromorphic form in $z$ with poles at $w$ and $P$. It is the third order abelian differential normalized by requiring that its integrals around the $A_{I}$ curves chosen to cut open the surface be zero.

We can now return to correlation functions of scalar fields. The two point function $G(z, w)=\langle x(z) x(w)\rangle$ satisfies the equations

$$
\begin{aligned}
& \partial_{z} \partial_{\bar{z}} G(z, w)=-2 \pi \delta(z-w)+\frac{2 \pi g_{z \bar{z}}}{\int d^{2} z \sqrt{g}}, \\
& \partial_{z} \partial_{\bar{w}} G(z, w)=2 \pi \delta(z-w)-\pi \sum_{I, J=1}^{h} \omega_{I}(z)(\operatorname{Im} \Omega)_{I J}^{-1} \bar{\omega}_{J}(w),
\end{aligned}
$$

and is clearly neither conformally invariant nor holomorphically split in terms of the moduli parameter $\Omega$ and the insertion points $z$ and $w$. The lack of conformal invariance reflects the fact that $x$ is not a well defined conformal field with a definite dimension. On the other hand vertex operators

$$
V_{k}(z)=\left(2 g_{z \bar{z}}\right)^{k^{2} / 2}: e^{i k_{\mu} x^{\mu}(z)}:
$$

have well-defined conformal dimensions $\left(k^{2} / 2, k^{2} / 2\right)$. Their correlation functions can be written as

$$
\left\langle\prod_{i=1}^{n} V_{k_{i}}\left(z_{i}\right)\right\rangle=(2 \pi)^{10} \delta(k) \prod_{i<j} F\left(z_{i}, z_{i}\right)^{k_{i} \cdot k_{j}}
$$


where $k=\sum k_{i}$ and $F(z, w)$ is defined by

$$
F(z, w)=\left(2 g_{z \bar{z}} g_{w \bar{w}}\right)^{-1 / 2} \exp \left(-G(z, w)+\frac{1}{2} G_{R}(z, z)+\frac{1}{2} G_{R}(w, w)\right)
$$

with $G_{R}(z, z)$ the reparametrization invariant regularized Green's function at coincident points. The function $F(z, w)$, though conformally invariant, can still not be split into a holomorphic and an anti-holomorphic part. Rather it admits the following expression in terms of the prime form

$$
F(z, w)=|E(z, w)|^{2} \exp \left(-2 \pi \operatorname{Im} \int_{w}^{z} \omega_{I}(\operatorname{Im} \Omega)_{I J}^{-1} \operatorname{Im} \int_{w}^{z} \omega_{J}\right) .
$$

The second factor on the right-hand side is typical of many obstructions to holomorphic splitting we shall encounter. From (2.17) and (2.18) we can isolate those pieces of the Green's function $G(z, w)$ which are both conformally invariant and holomorphically split:

$$
G(z, w)=-\ln E(z, w)-\ln \overline{E(z, w)}+2 \pi \operatorname{Im} \int_{w}^{z} \omega_{I}(\operatorname{Im} \Omega)_{I J}^{-1} \operatorname{Im} \int_{w}^{z} \omega_{J}+f(z)+f(w),
$$

where

$$
f(z)=\frac{1}{2} G_{R}(z, z)-\frac{1}{2} \ln g_{z \bar{z}} .
$$

The multiple-valued functions $-\ln E(z, w)$ and $-\ln \overline{E(z, w)}$ can be viewed as effective Green's functions for chiral scalar fields $x_{+}$and $x_{-}$. The multiplevaluedness will disappear from physical amplitudes. For such amplitudes we may use the symbolic rule

$$
\partial_{z} \partial_{\bar{z}} \ln E(z, w)=2 \pi \delta(z-w) .
$$

Finally we note that the expectation value of the chiral stress tensor can also be derived from the prime form

$$
\begin{gathered}
-\frac{4 \pi}{\sqrt{g}} \frac{\delta}{\delta g^{z \bar{z}}} \ln Z_{\Delta}=\left\langle T_{z z}\right\rangle, \\
\left\langle T_{z z}\right\rangle=-\frac{1}{2} \lim _{z \rightarrow w}\left(\partial_{z} \partial_{w} \ln E(z, w)-\frac{1}{(z-w)^{2}}\right) .
\end{gathered}
$$

\section{Scalar Superfields on a Super Riemann Surface}

In this section we provide a brief description of scalar superfields on super Riemann surfaces in the supergravity formalism $[13,24]$.

Local two-dimensional superspace is parametrized by coordinates $(\xi, \bar{\xi}, \theta, \bar{\theta})$. The supergravity fields are a superzweibein $E_{M}{ }^{A}$ and a $U(1)$ connection $\Omega_{M}$ satisfying the Wess-Zumino constraints. If we denote by ${ }^{1}$

$$
\mathscr{D}_{A}^{(n)}=E_{A}{ }^{M}\left(\partial_{M}+i n \Omega_{M}\right)
$$

\footnotetext{
${ }^{1}$ The index $A$ stands for $A=(a, \alpha)$, where $a$ is the $U(1)$ vector index and $\alpha$ is the $U(1)$ spinor index
} 
the superderivatives on $U(1)$ tensors of rank $n$, and define the torsion and curvature tensors by

$$
\left\{\mathscr{D}_{A}, \mathscr{D}_{B}\right\}=T_{A B}{ }^{c} \mathscr{D}_{C}+i n R_{A B},
$$

then the constraints are

$$
T_{a b}{ }^{c}=T_{\alpha \beta}{ }^{\gamma}=0, \quad T_{\alpha \beta}{ }^{c}=2 \gamma_{\alpha \beta}^{c} .
$$

They are invariant under local $U(1)$ transformations, local super-Weyl rescalings, and super-reparametrizations. An equivalence class under these transformations of supergeometries satisfying the Wess-Zumino constraints is a super Riemann surface.

The unique action for a scalar superfield $X^{\mu}$ consistent with the above symmetries, as well as space-time Poincare symmetry is given by

$$
I_{m}\left(E_{M}{ }^{A}, X^{\mu}\right)=\frac{1}{4 \pi} \int d^{2} \mathbf{z} E \mathscr{D}_{+} X^{\mu} \mathscr{D}_{-} X^{\mu}+\lambda \chi(M),
$$

where $\chi(M)$ is the Euler characteristic, and $E=s \operatorname{det} E_{M}{ }^{A}$.

In superstring theory the history of the string is given by a supergeometry and 10 scalar superfields $X^{\mu}$ which describe the imbedding of the super surface into 10-dimensional space-time. Summing over histories means that both scalar superfields and supergeometries have to be quantized. As explained in the introduction, the difficulties in constructing chiral amplitudes are however entirely due to the scalar superfields. This is why we have concentrated on quantizing these fields only, viewing the supergeometry as a fixed background. Quantization of supergeometries will be taken up again only in Sect. 7 and thereafter.

In a theory of scalar superfields correlation functions are of the form

$$
\left\langle\prod_{i=1}^{n} V_{i}\left(\mathbf{z}_{i}\right)\right\rangle_{X}=\int D X^{\mu} \prod_{i=1}^{n} V_{i}\left(\mathbf{z}_{i}\right) e^{-I_{m}},
$$

where the measure and the operators $V_{i}\left(\mathbf{z}_{i}\right)$ are constrained by invariance under $s$ Diff, $s$ Weyl, and local $U(1)$. It is well-known that $s$ Weyl is anomalous. In practice we shall deal with situations where the $s$ Weyl anomalies of the scalar superfields are cancelled by other fields, and we can thus ignore them. This is for example the case in superstring theory, where the anomalies of the 10 scalar superfields cancel those of the superghosts. The $\mathbf{z}_{i}=\left(z_{i}, \theta_{i}\right)$ denote insertion points, which are just $n$ distinct points on the super Riemann surface.

We shall restrict our attention to those vertices only which can later represent physical particles in the superstring spectrum. Rules for identifying such vertices have been enunciated in [23]. Here we just note that Poincare symmetry in the space-time index $\mu$ and $s$ Diff invariance dictate that they be of the form

$$
V_{k}(\mathbf{z})=E P\left(\mathscr{D}_{+} X^{\mu}, \mathscr{D}_{-} X^{\mu}, \varepsilon\right) e^{i k_{\mu} X^{\mu}},
$$

where $P$ is a polynomial in the covariant derivatives and derivatives thereof, and is linear in the polarization tensor $\varepsilon$. Of course there are further conditions on the precise form of $V_{k}$ due to local $U(1)$ and $s$ Weyl invariance which imply in 
particular that the masses of these particles must be $m^{2}=-k_{\mu} k^{\mu}=0,2,4, \ldots$ Of central interest to us are the vertices for massless bosons, which are rather simple and given by

$$
V_{k}=\varepsilon_{\mu \bar{\mu}} E \mathscr{D}_{+} X^{\mu} \mathscr{D}_{-} X^{\bar{\mu}} e^{i k_{\mu} X^{\mu}} .
$$

They can be obtained by selecting the $\zeta^{\mu} \bar{\zeta}^{\bar{\mu}}$ coefficient in the generating function

$$
V(k, \mathbf{z}, \overline{\mathbf{z}} ; \zeta, \bar{\zeta})=\exp \left[i k_{\mu} X^{\mu}(\mathbf{z})+\zeta^{\mu} \sqrt{E} \mathscr{D}_{+} X^{\mu}(\mathbf{z})+\bar{\zeta}^{\bar{\mu}} \sqrt{E} \mathscr{D}_{-} X^{\bar{\mu}}(\mathbf{z})\right] .
$$

Thus our main task is to analyze the holomorphic structure of the following correlation function

$$
\left\langle\prod_{i=1}^{n} V\left(k_{i} ; \mathbf{z}_{i}, \overline{\mathbf{z}}_{i} ; \zeta_{i}, \bar{\zeta}_{i}\right)\right\rangle_{X}
$$

as a function of the background supergeometry, the polarization tensors $\zeta_{i}$, and the insertion points $\mathbf{z}_{i}=\left(z_{i}, \theta_{i}\right)$.

We shall carry out quantization in component language, and at the end regroup the answer in terms of super-analytic objects. The passage from superfields to components is achieved by going to Wess-Zumino gauge, where the fields of supergeometry can be expressed in terms of an ordinary zweibein $e_{m}{ }^{a}$, a world-sheet gravitino $\chi_{m}{ }^{\alpha}$, and an auxiliary field $A$ (see, e.g., [24, 13]). In the critical dimension super-Weyl invariance allows to set $\gamma^{m} \chi_{m}=0$, so that only the components $\chi_{\bar{z}}^{+}$and $\chi_{z}^{-}$of the gravitino survive. Similarly $A$ may be set to 0 . The scalar superfield and its superderivatives are then given by

$$
\begin{aligned}
X^{\mu}(\mathbf{z})= & x^{\mu}(z)+\theta \psi_{+}^{\mu}(z)+\bar{\theta} \psi_{-}^{\mu}(z)+i \theta \bar{\theta} F^{\mu}(z), \\
\mathscr{D}_{-} X^{\mu}= & \psi_{-}^{\mu}-i \theta F^{\mu}+\bar{\theta}\left(D_{\bar{z}} x^{\mu}+\frac{1}{2} \chi_{\bar{z}}^{+} \psi_{+}^{\mu}\right) \\
& +\theta \bar{\theta}\left(-D_{\bar{z}} \psi_{+}^{\mu}-\frac{1}{2} \chi_{\bar{z}}^{+} D_{z} x^{\mu}-\frac{1}{4} \chi_{\bar{z}}^{+} \chi_{z}^{-} \psi_{-}^{\mu}\right) .
\end{aligned}
$$

The superfield action of (3.4) is easily evaluated in components and we recover the standard RNS action,

$$
\begin{aligned}
I_{m}= & \frac{1}{4 \pi} \int d^{2} z e\left[D_{z} x^{\mu} D_{\bar{z}} x^{\mu}-\psi_{+}^{\mu} D_{\bar{z}} \psi_{+}^{\mu}-\psi_{-}^{\mu} D_{z} \psi_{-}^{\mu}+F^{\mu} F^{\mu}\right. \\
& \left.+\chi_{\bar{z}}^{+} \psi_{+}^{\mu} D_{z} x^{\mu}+\chi_{\bar{z}}^{-} \psi_{-}^{\mu} D_{\bar{z}} x^{\mu}-\frac{1}{2} \chi_{\bar{z}}^{+} \chi_{\bar{z}}^{-} \psi_{+}^{\mu} \psi_{-}^{\mu}\right] .
\end{aligned}
$$

The covariant derivatives acting on $U(1)$ tensors of rank $n$ are

$$
D_{z}^{(n)}=e_{z}{ }^{m}\left(\partial_{m}+i n \omega_{m}\right)
$$

with the spin connection given by

$$
\omega_{m}=-e_{m}{ }^{a} \varepsilon^{p q} \partial_{p} e_{q}^{b} \delta_{a b} .
$$

The symmetries of the theory are now reduced to those that fix Wess-Zumino gauge. They translate into reparametrization invariance, Weyl scalings, and local 
supersymmetry. The infinitesimal forms of the first two symmetries are wellknown. The last one acts in the following way:

$$
\begin{aligned}
\delta x^{\mu} & =\zeta^{+} \psi_{+}^{\mu}+\zeta^{-} \psi_{-}^{\mu}, \\
\delta \psi_{+}^{\mu} & =\zeta^{+}\left(D_{z} x^{\mu}+\frac{1}{2} \chi_{z}^{-} \psi_{-}^{\mu}\right)-i \zeta_{+} F^{\mu}, \\
\delta F^{\mu} & =\frac{i}{2}\left(\chi_{p} \gamma^{m} \gamma^{p} \zeta\right)\left(\partial_{m} x^{\mu}+\frac{1}{2} \chi_{m} \psi^{\mu}\right)-i \zeta \gamma^{m} D_{m} \psi^{\mu}, \\
\delta e_{m}{ }^{z} & =-\zeta^{+} \chi_{m}^{+}, \quad \delta \chi_{\bar{z}}^{+}=-2 D_{\bar{z}} \zeta^{+} .
\end{aligned}
$$

Although the auxiliary field $F^{\mu}$ is often discarded from the start, we shall keep it for the time being. This guarantees that local supersymmetry is kept linear. The contractions of auxiliary fields give rise to contact terms which we shall discuss in Appendix B.

\section{Chiral Amplitudes for Scalar Superfields: Preliminary Version}

In this section we shall obtain first a version of chiral amplitudes which is not yet manifestly supersymmetric. We consider non-chiral amplitudes, and both chiralities are endowed with the same spin structure $\delta$. The goal of chiral splitting is to separate not only opposite chiralities, but also holomorphic and anti-holomorphic dependence on moduli, world-sheet gravitino field $\chi_{\bar{z}}^{+}$, chiral polarization tensors, and vertex insertion points.

Even ignoring all global issues on the world-sheet such as zero modes, we cannot - even naively - expect to obtain such splitting as things stand. The reason is that the exponential factor $\exp (i k X)$ in $(3.6-3.9)$ for real $k$ is not even formally the product of an operator by its complex conjugate. Let indeed $X=X_{+}+X_{-}$ with

$$
X_{+}=x_{+}+\theta \psi_{+}, \quad X_{-}=x_{-}+\bar{\theta} \psi_{-} .
$$

Then

$$
\exp (i k X)=\exp \left(i k X_{+}\right) \exp \left(i k X_{-}\right)
$$

and the factors on the right-hand side are not complex conjugates of one another. Clearly this can be remedied by analytically continuing $k$ to purely imaginary values, so that the vertex operators are at least formally split. For massless particles, this is certainly consistent with the mass-shell condition, but in general this analytic continuation should only be viewed as a useful trick in exhibiting the chiral and holomorphic splitting of string amplitudes. The remaining contributions to the vertex operators in (3.8) are already formally split provided one views $\bar{\zeta}$ as the complex conjugate of $\zeta$. Consistency requires that the internal loop momenta which appear later be continued to purely imaginary values as well. Henceforth we assume that this has been done.

It is convenient to rewrite the amplitudes under consideration

$$
\mathscr{A}_{\delta}=\left\langle\prod_{i=1}^{n} V\left(k_{i} ; \mathbf{z}_{i}, \overline{\mathbf{z}}_{i} ; \zeta_{i}, \bar{\zeta}_{i}\right)\right\rangle=\int D X^{\mu} e^{I_{m}} \prod_{i=1}^{n} V\left(k_{i} ; \mathbf{z}_{i}, \overline{\mathbf{z}}_{i} ; \zeta_{i}, \bar{\zeta}_{i}\right)
$$


as

$$
\mathscr{A}_{\delta}=\int D \psi_{+}^{\mu} D \psi_{-}^{\mu} e^{-I_{0}(\psi+)-I_{0}(\psi-)} e^{S_{\psi}+\bar{S}_{\psi}+T_{\psi}} \mathscr{A}_{x} \mathscr{A}_{F},
$$

where $\mathscr{A}_{x}$ and $\mathscr{A}_{F}$ are the amplitudes resulting from the $x^{\mu}$ and $F^{\mu}$ contributions:

$$
\begin{aligned}
& \mathscr{A}_{x}=\int D x^{\mu} e^{-I_{0}\left(x^{\mu}\right)} \prod_{i=1}^{n} e^{i k_{i}^{\mu} x_{\mu}\left(z_{i}\right)} e^{S_{x}}, \\
& \mathscr{A}_{F}=\int D F^{\mu} e^{S_{F}} .
\end{aligned}
$$

The actions $I_{0}$ are the purely quadratic actions for the corresponding fields, and the interaction terms $S_{x}, S_{F}, S_{\psi}$, and $T_{\psi}$ are given by

$$
\begin{aligned}
& S_{x}=-\frac{1}{4 \pi} \int d^{2} z \chi_{\bar{z}}^{+} \psi_{+}^{\mu} \partial_{z} x^{\mu}-\frac{1}{4 \pi} \int d^{2} z \chi_{z}^{-} \psi_{-}^{\mu} \partial_{\bar{z}} x^{\mu}+\frac{1}{8 \pi} \int d^{2} z \chi_{\bar{z}}^{+} \chi_{z}^{-} \psi_{+}^{\mu} \psi_{-}^{\mu} \\
&+\sum_{i=1}^{n}\left(\zeta_{i}^{\mu} \theta_{i} \partial_{z} x^{\mu}\left(z_{i}\right)+\frac{1}{2} \bar{\zeta}_{i} \bar{\theta}_{i} \theta_{i} \chi_{\bar{z}}^{+}\left(z_{i}\right) \partial_{z} x\left(z_{i}\right)+\text { c.c. }\right) \\
& S_{F}=- \frac{1}{4 \pi} \int d^{2} z e F_{\mu} F^{\mu}+\sum_{i=1}^{n}\left(-\theta_{i} \bar{\theta}_{i} k_{i}^{\mu} F^{\mu}\left(z_{i}\right)+i \zeta_{i}^{\mu} \bar{\theta}_{i} F^{\mu}\left(z_{i}\right)-i \bar{\zeta}_{i}^{\mu} \theta_{i} F^{\mu}\left(z_{i}\right)\right), \\
& S_{\psi}=\sum_{i=1}^{n}\left(i k_{i}^{\mu} \theta_{i} \psi_{+}^{\mu}\left(z_{i}\right)+\zeta_{i}^{\mu} \psi_{+}^{\mu}\left(z_{i}\right)-\bar{\zeta}_{i}^{\mu} \theta_{i} \bar{\theta}_{i} D_{\bar{z}} \psi_{+}^{\mu}\left(z_{i}\right)+\text { c.c. }\right) \\
& T_{\psi}=\sum_{i=1}^{n}\left(-\frac{1}{8} \zeta_{i}^{\mu} \theta_{i} \bar{\theta}_{i} \chi_{\bar{z}}^{+} \chi_{z}^{-} \psi_{+}^{\mu}\left(z_{i}\right)+\frac{1}{2} \bar{\zeta}_{i}^{\mu} \bar{\theta}_{i} \chi_{\bar{z}}^{+} \psi_{+}^{\mu}\left(z_{i}\right)+\text { c.c. }\right) .
\end{aligned}
$$

\section{Contractions of $F^{\mu}$}

Contractions of the auxiliary fields $F^{\mu}$ are independent of the other fields and evaluated in Appendix B, where it is argued that they can be dropped from the final answer for scattering amplitudes.

\section{Contractions of $x^{\mu}$ Fields}

Our first task is to obtain a more tractable expression for the amplitude $\mathscr{A}_{x}$ in (4.3). Rewriting the $S_{x}$ term of (4.4) as

$$
S_{x}=\frac{1}{8 \pi} \int d^{2} z \chi_{\bar{z}}^{+} \chi_{\bar{z}}^{-} \psi_{+}^{\mu} \psi_{-}^{\mu}+\int d^{2} z \bar{\zeta}_{z}^{\mu} \partial_{\bar{z}} x^{\mu}+\int d^{2} z \zeta \partial_{z} x^{\mu}
$$

with

$$
\zeta_{\bar{z}}^{\mu}(z)=-\frac{1}{4 \pi} \chi_{\bar{z}}^{+} \psi_{+}^{\mu}(z)+\sum_{i=1}^{n} \delta\left(z-z_{i}\right)\left(\zeta_{i}^{\mu} \theta_{i}+\frac{1}{2} \bar{\zeta}_{i}^{\mu} \bar{\theta}_{i} \theta_{i} \chi_{\bar{z}}^{+}\left(z_{i}\right)\right)
$$

and $\bar{\zeta}_{z}^{\mu}(z)$ its complex conjugate, we obtain in view of the rules for bosonic scalar contractions of Sect. 2,

$$
\mathscr{A}_{x}=(2 \pi)^{10} \delta(k)\left[\frac{8 \pi^{2} \operatorname{det}^{\prime} \Delta}{\operatorname{det} \operatorname{Im} \Omega \int d^{2} z \sqrt{g}}\right]^{-5}(\operatorname{det} \operatorname{Im} \Omega)^{-5} e^{\mathscr{K}^{0}+\mathscr{K}^{1}+\mathscr{K}^{2}} .
$$


Here the terms $\mathscr{K}^{i}$ are of degrees $i$ in $\zeta_{\bar{z}}^{\mu}$,

$$
\begin{aligned}
\mathscr{K}^{0}= & -\sum_{i<j} k_{i}^{\mu} k_{j}^{\mu} G\left(z_{i}, z_{j}\right), \\
\mathscr{K}^{1}= & \sum_{i=1}^{n} i k_{i}^{\mu} \int d^{2} z \bar{\zeta}_{z}^{\mu} \partial_{\bar{z}} G\left(z, z_{i}\right)+i k_{i}^{\mu} \int d^{2} z \zeta_{\bar{z}}^{\mu} \partial_{z} G\left(z, z_{i}\right), \\
\mathscr{K}^{2}= & \frac{1}{8 \pi} \int d^{2} z \chi_{\bar{z}}^{+} \chi_{z}^{-} \psi_{+}^{\mu} \psi_{-}^{\mu}+\int d^{2} z d^{2} w \bar{\zeta}_{z}^{\mu} \zeta_{\bar{w}}^{\mu} \partial_{\bar{z}} \partial_{w} G(z, w) \\
& +\frac{1}{2} \int d^{2} z d^{2} w \bar{\zeta}_{z}^{\mu} \bar{\zeta}_{w}^{\mu} \partial_{\bar{z}} \partial_{\bar{w}} G(z, w) \\
& +\frac{1}{2} \int d^{2} z d^{2} w \zeta_{\bar{z}}^{\mu} \zeta_{\bar{w}}^{\mu} \partial_{z} \partial_{w} G(z, w) .
\end{aligned}
$$

Now the combination involving the determinant of the Laplacian in (4.7) can be considered as split, since it equals $\left|Z_{\Delta}(\Omega)\right|^{-20}$ up to a local holomorphic anomaly which will ultimately cancel in view of (2.2). Next we can rewrite everything else in terms of the effective chiral Green's functions $-\ln E(z, w)$ and $-\ln \overline{E(z, w)}$ of (2.16), and the imaginary part of the period matrix. This is because all the terms involving the non-scale invariant part $f(z)$ of the Green's functions $G(z, w)$ will cancel due to overall momentum conservation $\sum k_{i}^{\mu}=0$. The result is

$$
\mathscr{K}^{i}=\mathscr{L}_{+}^{i}+\mathscr{L}_{-}^{i}+\mathscr{L}^{i}, \quad i=0,1,2,
$$

where the $\mathscr{L}_{+}^{i}, \mathscr{L}_{-}^{i}$ 's are the terms involving the chiral Green's functions,

$$
\begin{aligned}
& \mathscr{L}_{+}^{0}=\sum_{i<j} k_{i}^{\mu} k_{j}^{\mu} \ln E\left(z_{i}, z_{j}\right), \\
& \mathscr{L}_{+}^{1}=-\sum_{i=1}^{n} i k_{i}^{\mu} \int d^{2} z \zeta_{\bar{z}}^{\mu} \partial_{z} \ln E\left(z, z_{i}\right), \\
& \mathscr{L}_{+}^{2}=-\frac{1}{2} \int d^{2} z d^{2} w \zeta_{\bar{z}}^{\mu} \zeta_{\bar{w}}^{\mu} \partial_{z} \partial_{w} \ln E(z, w),
\end{aligned}
$$

and the $\mathscr{L}^{i}$ 's are terms involving the imaginary part of $\Omega$,

$$
\begin{aligned}
\mathscr{L}^{0}= & 2 \pi \sum_{i<j} k_{i}^{\mu} k_{j}^{\mu} \operatorname{Im} \int_{P}^{z_{i}} \omega_{I}(\operatorname{Im} \Omega)_{I J}^{-1} \operatorname{Im} \int_{P}^{z_{j}} \omega_{J}, \\
\mathscr{L}^{1}= & -4 \pi i \sum_{i=1}^{n} k_{i}^{\mu} \operatorname{Im} \int d^{2} z \zeta_{\bar{z}}^{\mu} \omega_{I}(z)(\operatorname{Im} \Omega)_{I J}^{-1} \operatorname{Im} \int_{P}^{z_{l}} \omega_{J}, \\
\mathscr{L}^{2}= & -2 \pi \operatorname{Im} \int d^{2} z \zeta_{\bar{z}}^{\mu} \omega_{I}(z)(\operatorname{Im} \Omega)_{I J}^{-1} \operatorname{Im} \int d^{2} w \zeta_{\bar{w}}^{\mu} \omega_{J}(w) \\
& +\frac{1}{8 \pi} \int d^{2} z \chi_{\bar{z}}^{+} \chi_{z}^{-} \psi_{+}^{\mu} \psi_{-}^{\mu}+2 \pi \int d^{2} z \bar{\zeta}_{z}^{\mu} \zeta_{\bar{z}}^{\mu} .
\end{aligned}
$$

We have again exploited momentum conservation to arrive at the above expressions for $\mathscr{L}^{i}$ in terms of an arbitrary base point $P$. The last two terms in $\mathscr{L}^{2}$ 
lead to some drastic simplifications. In fact the quartic terms $\chi_{\bar{z}}^{+} \chi_{\bar{z}}^{-} \psi_{+}^{\mu} \psi_{-}^{\mu}$ immediately cancel as already noted in [25]. The other terms can be expanded as

$$
-\frac{1}{2} \sum_{i=1}^{n} \chi_{z}^{-} \psi_{-}^{\mu}\left(z_{i}\right)\left(\zeta_{i}^{\mu} \theta_{i}+\frac{1}{2} \bar{\zeta}_{i}^{\mu} \bar{\theta}_{i} \theta_{i} \chi_{\bar{z}}^{+}\left(z_{i}\right)\right)+\text { c.c. }
$$

plus a contact term which we ignore again in view of the considerations in Appendix B. This sum cancels precisely the non-split terms arising from the vertex operators, i.e., $T_{\psi}$. Actually there is a contribution arising from the passage from the super measure to the component form at this point that has to be taken into account. The remaining terms in $\mathscr{L}^{i}$ now all involve $(\operatorname{Im} \Omega)^{-1}$ in the same way and can be recast as

$$
\exp \left(\mathscr{L}^{0}+\mathscr{L}^{1}+\mathscr{L}^{2}+T_{\psi}\right)=\exp \left(\frac{\pi}{2}\left(\zeta_{I}^{\mu}-\bar{\zeta}_{I}^{\mu}\right)(\operatorname{Im} \Omega)_{I J}^{-1}\left(\zeta_{J}^{\mu}-\bar{\zeta}_{J}^{\mu}\right)\right)
$$

with

$$
\zeta_{I}^{\mu}=\int d^{2} z \zeta_{\bar{z}}^{\mu} \omega_{I}(z)+i \sum_{i=1}^{n} k_{i}^{\mu} \int_{P}^{z_{i}} \omega_{I}
$$

We observe that the fields $\zeta_{\bar{z}}^{\mu}$ involve fermionic fields of one definite chirality only (although they do not depend holomorphically on insertion points). Thus a first form of chiral splitting can be achieved if we could separate $\zeta_{\bar{z}}^{\mu}$ from $\bar{\zeta}_{z}^{\mu}$. Remarkably, the expression in (4.13) can be split at fixed internal momenta $p_{I}^{\mu}$. More precisely

$(\operatorname{det} \operatorname{Im} \Omega)^{-5} \exp \left(\mathscr{L}^{0}+\mathscr{L}^{1}+\mathscr{L}^{2}+T_{\psi}\right)=\int_{\mathscr{T}} d p_{I}^{\mu}\left|\exp \left(i \pi p_{I}^{\mu} \Omega_{I J} p_{J}^{\mu}+2 \pi p_{I}^{\mu} \zeta_{I}^{\mu}\right)\right|^{2}$.

The subscript $\mathscr{T}$ indicates that the absolute value is taken with $p_{I}^{\mu}$ and $k_{i}^{\mu}$ purely imaginary, after which the result is analytically continued to real values and then integrated over. The motivation for this prescription was given at the beginning of this section. We have in this way arrived at a form where the contributions $\mathscr{F}_{\delta}^{m}\left(p_{I}^{\mu}\right), \overline{\mathscr{F}}_{\delta}^{m}\left(p_{I}^{\mu}\right)$ of fields of opposite chiralities can be recognized in the chirally symmetric amplitude

with

$$
\mathscr{A}_{\delta}=(2 \pi)^{10} \delta(k) \int_{\mathscr{T}} d p_{I}^{\mu}\left|\mathscr{F}_{\delta}^{m}\left(p_{I}^{\mu}\right)\right|^{2}
$$

$$
\mathscr{F}_{\delta}^{m}\left(p_{I}^{\mu}\right)=Z_{\Delta}(\Omega)^{-10} e^{i \pi p_{I}^{\mu} \Omega_{I J} p_{J}^{\mu}} \int D \psi_{+}^{\mu} e^{\mathscr{L}_{+}^{0}+\mathscr{L}_{+}^{1}+\mathscr{L}_{+}^{2}-I_{0}\left(\psi_{+}\right)+S_{\psi}+2 \pi p_{I}^{\mu} \zeta_{T}^{\mu}} .
$$

As usual we omit the contribution of the Weyl anomalies.

\section{Shifting of the $\psi_{+}$Integrals}

Our next objective is to exhibit the holomorphic dependence on insertion points and polarization tensors. To isolate the non-split terms, we decompose the various 
factors in (4.15) and (4.16):

$$
\begin{aligned}
& \mathscr{L}_{+}^{0}=\mathscr{L}_{++}^{0}=\sum_{i<j} k_{i}^{\mu} k_{j}^{\mu} \ln E\left(z_{i}, z_{j}\right), \\
& \mathscr{L}_{+}^{1}=\mathscr{L}_{++}^{1}+\mathscr{L}_{+-}^{1}, \quad \mathscr{L}_{+}^{2}=\mathscr{L}_{++}^{2}+\mathscr{L}_{+-}^{2}, \\
& S_{\psi}=S_{\psi,+}+S_{\psi,-}, \quad \zeta_{I}^{\mu}=\zeta_{I,+}^{\mu}+\zeta_{I,-}^{\mu},
\end{aligned}
$$

with the holomorphic contributions given by

$$
\begin{aligned}
\mathscr{L}_{++}^{1}= & \frac{i}{4 \pi} \sum_{i=1}^{n} k_{i}^{\mu} \int d^{2} z \chi_{\bar{z}}^{+} \psi_{+}^{\mu} \partial_{z} \ln E\left(z, z_{i}\right)-i \sum_{i, j} k_{i}^{\mu} \zeta_{j}^{\mu} \theta_{j} \partial_{z_{j}} \ln E\left(z_{i}, z_{j}\right), \\
\mathscr{L}_{++}^{2}= & -\frac{1}{32 \pi^{2}} \int d^{2} z d^{2} w \chi_{\bar{z}}^{+} \psi_{+}^{\mu} \partial_{z} \partial_{w} \ln E(z, w) \chi_{\bar{w}}^{+} \psi_{+}^{\mu}(w) \\
& +\sum_{i} \frac{1}{4 \pi} \int d^{2} z \chi_{\bar{z}}^{+} \psi_{+}^{\mu} \partial_{z} \partial_{z_{i}} \ln E\left(z, z_{i}\right) \zeta_{i}^{\mu} \theta_{i} \\
& -\frac{1}{2} \sum_{i j} \zeta_{i}^{\mu} \theta_{i} \partial_{z_{i}} \partial_{z_{J}} \ln E\left(z_{i}, z_{j}\right) \zeta_{j}^{\mu} \theta_{j} \\
S_{\psi,+}= & \sum_{i=1}^{n}\left(i k_{i}^{\mu} \theta_{i} \psi_{+}^{\mu}\left(z_{i}\right)+\zeta_{i}^{\mu} \psi_{+}^{\mu}\left(z_{i}\right)\right), \\
\zeta_{I,+}^{\mu}= & -\frac{1}{4 \pi} \int d^{2} z \chi_{\bar{z}}^{+} \psi_{+}^{\mu} \omega_{I}+\sum_{i}\left(i k_{i}^{\mu} \int_{P}^{z_{i}} \omega_{I}+\zeta_{i}^{\mu} \theta_{i} \omega_{I}\left(z_{i}\right)\right)
\end{aligned}
$$

and the non-holomorphic terms by

$$
\begin{aligned}
\mathscr{L}_{+-}^{1}= & \frac{i}{2} \sum_{i, j} k_{i}^{\mu} \theta_{j} \bar{\theta}_{j} \bar{\zeta}_{j}^{\mu} \chi_{\bar{z}}^{+}\left(z_{j}\right) \partial_{z_{j}} \ln E\left(z_{i}, z_{j}\right), \\
\mathscr{L}_{+-}^{2}= & -\frac{1}{8 \pi} \int d^{2} z \sum_{i=1}^{n} \chi_{\bar{z}}^{+} \psi_{+}^{\mu} \partial_{z} \partial_{z_{i}} \ln E\left(z, z_{i}\right) \theta_{i} \bar{\theta}_{i} \bar{\zeta}_{i}^{\mu} \chi_{\bar{z}}^{+}\left(z_{i}\right) \\
& -\frac{1}{8} \sum_{i j} \theta_{i} \bar{\theta}_{i} \theta_{j} \bar{\theta}_{j} \bar{\zeta}_{i}^{\mu} \chi_{\bar{z}}^{+}\left(z_{i}\right) \bar{\zeta}_{j}^{\mu} \chi_{\bar{z}}^{+}\left(z_{j}\right) \partial_{z_{i}} \partial_{z_{J}} \ln E\left(z_{i}, z_{j}\right) \\
& +\frac{1}{2} \sum_{i, j} \zeta_{i}^{\mu} \theta_{i} \theta_{j} \bar{\theta}_{j} \bar{\zeta}_{j}^{\mu} \chi_{\bar{z}}^{+}\left(z_{j}\right) \partial_{z_{i}} \partial_{z_{j}} \ln E\left(z_{i}, z_{j}\right), \\
S_{\psi,-}= & -\sum_{i=1}^{n} \bar{\zeta}_{i}^{\mu} \theta_{i} \bar{\theta}_{i} D_{\bar{z}} \psi_{+}^{\mu}\left(z_{i}\right), \\
\zeta_{I,-}^{\mu}= & \frac{1}{2} \sum_{i} \bar{\zeta}_{i}^{\mu} \bar{\theta}_{i} \theta_{i} \chi_{\bar{z}}^{+}\left(z_{i}\right) \omega_{I}\left(z_{i}\right) .
\end{aligned}
$$

It is a remarkable fact that the non-holomorphic contributions $\mathscr{L}_{+-}^{1}, \mathscr{L}_{+-}^{2}, S_{\psi,-}$ and $\zeta_{I,-}^{\mu}$ - completely cancel out of the final answer. To see this, it is necessary to carry out all contractions of fermion fields occurring within the non-holomorphic 
contributions of (4.19). A key feature is the emergence of an additional quadratic term in $\psi_{+}$at this stage, which is the first term in the expression for $\mathscr{L}_{++}^{2}$ above. It can be characterized by the kernel

$$
K(z, w)=\frac{1}{8 \pi} \chi_{\bar{z}}^{+} \chi_{\bar{w}}^{+} \partial_{z} \partial_{w} \ln E(z, w)
$$

and leads to a modified Dirac operator

$$
\hat{\partial}_{\bar{z}} \psi_{+}=D_{\bar{z}} \psi_{+}+\int d^{2} w K(z, w) \psi_{+}(w) .
$$

We note that the kernel $K$ actually has only a simple pole due to its anti-symmetry, so that the integral defining $\hat{\partial}_{\bar{z}}$ is absolutely convergent. With this new operator we can single out the sources in the $\psi_{+}$dependent terms of (4.16), (4.18), and (4.19)

$$
S=\frac{1}{4 \pi} \int d^{2} z \psi_{+}^{\mu} \hat{\partial}_{\bar{z}} \psi_{+}^{\mu}+\int d^{2} z J^{\mu} \psi_{+}^{\mu}+\frac{1}{2 \pi} \int d^{2} z f^{\mu}(z) \hat{\partial}_{\bar{z}} \psi_{+}(z)
$$

with

$$
\begin{aligned}
J^{\mu}(z)= & \frac{i}{4 \pi} \sum_{i=1}^{n} k_{i}^{\mu} \chi_{\bar{z}}^{+} \partial_{z} \ln E\left(z, z_{i}\right)+\frac{1}{4 \pi} \sum_{i=1}^{n} \chi_{\bar{z}}^{+} \partial_{z} \partial_{z_{i}} \ln E\left(z, z_{i}\right) \zeta_{i}^{\mu} \theta_{i} \\
& +\sum_{i=1}^{n}\left(i k_{i}^{\mu} \theta_{i}+\zeta_{i}^{\mu}\right) \delta\left(z-z_{i}\right)-\frac{1}{2} \chi_{\bar{z}}^{+} \omega_{I}(z) p_{I}^{\mu}, \\
f^{\mu}(z)= & -2 \pi \sum_{i=1}^{n} \bar{\zeta}_{i}^{\mu} \theta_{i} \bar{\theta}_{i} \delta\left(z-z_{i}\right) .
\end{aligned}
$$

Shifting the $\psi_{+}^{\mu}$ fields by $-f^{\mu}$ yields

$$
S=\frac{1}{4 \pi} \int d^{2} z \psi_{+}^{\mu} \hat{\partial}_{\bar{z}} \psi_{+}^{\mu}-\frac{1}{4 \pi} \int d^{2} z f^{\mu} \hat{\partial}_{\bar{z}} f^{\mu}+\int d^{2} z J^{\mu} \psi_{+}^{\mu}-\int J^{\mu} f^{\mu} .
$$

It is easy to check that the last two integrals cancel precisely all the nonholomorphic parts in $\mathscr{L}$ listed in (4.18) and (4.19), up to contact terms. More precisely

$$
\begin{aligned}
\mathscr{F}_{\delta}^{m}= & Z_{\Delta}^{-10}(\Omega) \exp \left[i \pi p_{I}^{\mu} \Omega_{I J} p_{J}^{\mu}\right] \int D \psi_{+}^{\mu} \exp \left[-I_{0}\left(\psi_{+}\right)\right] \\
& \cdot \exp \left[\mathscr{L}_{++}^{0}+\mathscr{L}_{++}^{1}+\mathscr{L}_{++}^{2}+S_{\psi,+}+2 \pi p_{I}^{\mu} \zeta_{I,+}^{\mu}\right]
\end{aligned}
$$

We have then attained the main goal of this section, namely the entire chiral amplitude $\mathscr{F}_{\delta}^{m}\left(p_{I}^{\mu}\right)$ of (4.16) is an integral over fields of definite chirality, and all terms mixing holomorphic and anti-holomorphic external parameters have been eliminated. It is useful to recast the cumbersome effective actions $\mathscr{L}_{++}^{1,2}$ and $S_{\psi,+}$ in terms of contractions of a chiral scalar field $x_{+}$,

$$
\begin{aligned}
\mathscr{F}_{\delta}^{m}= & Z_{\Delta}(\Omega)^{-10} \exp \left[i \pi p_{I}^{\mu} \Omega_{I J} p_{J}^{\mu}+2 \pi p_{I}^{\mu} \sum_{i=1}^{n}\left(i k_{i}^{\mu} \int_{P}^{z_{i}} \omega_{I}+\zeta_{i}^{\mu} \theta_{i} \omega_{I}\left(z_{i}\right)\right)\right] \\
& \times \int D \psi_{+}^{\mu} e^{-I_{0}(\psi+)}\left\langle\exp \left[-\frac{1}{2} p_{I}^{\mu} \int d^{2} z \chi_{\bar{z}}^{+} \psi_{+}^{\mu} \omega_{I}-\frac{1}{4 \pi} \int d^{2} z \chi_{\bar{z}}^{+} \psi_{+}^{\mu} \partial_{z} x_{+}^{\mu}\right]\right. \\
& \left.\times \prod_{i=1}^{n} \exp \left[i k_{i}^{\mu}\left(x_{+}^{\mu}\left(z_{i}\right)+\theta_{i} \psi_{+}^{\mu}\left(z_{i}\right)\right)+\zeta_{i}^{\mu}\left(\psi_{+}^{\mu}\left(z_{i}\right)+\theta_{i} \partial_{z_{l}} x_{+}^{\mu}\left(z_{i}\right)\right)\right]\right\rangle
\end{aligned}
$$


where the $x_{+}$propagator is given by

$$
\begin{aligned}
& \left\langle\prod_{i=1}^{n} e^{i k_{i} x_{+}\left(z_{\imath}\right)}\right\rangle=\prod_{i<j} E\left(z_{i}, z_{j}\right)^{k_{i} k_{j}}, \\
& \left\langle x_{+}(z) x_{+}(w)\right\rangle=-\ln E(z, w) .
\end{aligned}
$$

An equivalent formula which is more suggestive from certain points of view is obtained by also re-expressing $\zeta_{I,+}^{\mu}$ in terms of $x_{+}$contractions, and representing the period matrix by iterated contour integrals, with the help of formula (2.11)

$$
\begin{aligned}
\mathscr{F}_{\delta}^{m}= & Z_{\Delta}(\Omega)^{-10} \int D \psi_{+}^{\mu} e^{-I_{0}(\psi+)}\left\langle\exp \left[i p_{I}^{\mu} \oint_{B_{I}} d z \partial_{z} x_{+}^{\mu}-\frac{1}{4 \pi} \int d^{2} z \chi_{\bar{z}}^{+} \psi_{+}^{\mu} \partial_{z} x_{+}^{\mu}\right]\right. \\
& \left.\times \prod_{i=1}^{n} \exp \left[i k_{i}^{\mu}\left(x_{+}^{\mu}\left(z_{i}\right)+\theta_{i} \psi_{+}^{\mu}\left(z_{i}\right)\right)+\zeta_{i}^{\mu}\left(\psi_{+}^{\mu}\left(z_{i}\right)+\theta_{i} \partial_{i} x_{+}^{\mu}\left(z_{i}\right)\right)\right]\right\rangle_{x_{+}} \cdot
\end{aligned}
$$

To complete our prescriptions, we still need to specify the fermionic propagators. As noted in the introduction, this issue requires some care. Also the above prescriptions are not yet written in terms of super-analytic notions, so that local supersymmetry is not manifest. This will be our next task. The analysis is rather different for even and odd spin structures, and we shall discuss the two cases separately.

\section{Superabelian Differentials, Superperiod Matrix, and Chiral Amplitudes for Even Spin Structures}

It is easy to see that for generic even spin structure $\delta$, the $\psi_{+}$functional integrals appearing in the prescriptions (4.25-4.28) for chiral amplitudes will be holomorphic in both moduli parameters and insertion points. In fact they will produce a chiral determinant and correlation functions of the Dirac operator on spinors of $U$ (1) rank 1/2. For generic even spin structure the Dirac operator has no zero mode. The correlation function of chiral fermions is then given by the Szego kernel

$$
\begin{aligned}
S_{\delta}(z, w) & =-\left\langle\psi_{+}(z) \psi_{+}(w)\right\rangle, \\
\partial_{\bar{z}} S_{\delta} & =2 \pi \delta(z-w) .
\end{aligned}
$$

In terms of the prime form it can be expressed as

$$
S_{\delta}=\frac{\vartheta[\delta]\left(\int_{w}^{z} \omega_{I}, \Omega\right)}{E(z, w) \vartheta[\delta](0, \Omega)},
$$

which is a meromorphic $1 / 2$ form in $z$ and $w$, with a single simple pole at $z=w$. It is also manifestly holomorphic as a function of the period matrix $\Omega$. As for the chiral determinant, the Belavin-Knizhnik theorem (1.1) guarantees that one can construct a holomorphic determinant from the one of the Laplacian $\Delta_{1 / 2}^{-}$,

$$
\operatorname{det} \Delta_{1 / 2}^{-}=\operatorname{det} D_{\bar{z}} \operatorname{det} D_{z}, \quad \operatorname{det} D_{\bar{z}}=Z_{\delta}(\Omega)^{2}
$$


up to a local anomaly which will cancel in the end. This immediately implies the holomorphicity properties of the amplitudes $\mathscr{F}_{\delta}^{m}$ we listed out earlier. We can now restate $\mathscr{F}_{\delta}^{m}$ in a more compact form using the chiral scalar superfield

$$
X_{+}(\mathbf{z})=x_{+}(z)+\theta \psi_{+}(z)
$$

in terms of which (4.26-4.28) become

$$
\begin{aligned}
\mathscr{F}_{\delta}^{m}= & Z_{\Delta}(\Omega)^{-10} Z_{\delta}(\Omega)^{10}\left\langle\operatorname { e x p } \left[-\frac{1}{4 \pi} \int d^{2} z \chi_{\bar{z}}^{+} \psi_{+}^{\mu} \partial_{z} x_{+}^{\mu}\right.\right. \\
& \left.\left.+i p_{I}^{\mu} \oint_{B_{I}} d z d \theta \partial_{+} X_{+}^{\mu}+i \sum_{i=1}^{n} k_{i}^{\mu} X_{+}^{\mu}\left(\mathbf{z}_{i}\right)+\sum_{i=1}^{n} \zeta_{i}^{\mu} \partial_{+} X_{+}^{\mu}(\mathbf{z})\right]\right\rangle .
\end{aligned}
$$

The contractions of $x_{+}$and $\psi_{+}$are to be carried out with the propagators of (4.27) and (5.1).

\section{Geometry of Super Riemann Surfaces with Even Spin Structures}

We shall now lay down the effective rules which will reproduce the chiral amplitudes just obtained in terms of the superconformal structure of the background supergeometry.

First we need the notion of super-abelian differentials $\hat{\omega}$, which are the forms of rank $1 / 2$ satisfying

$$
\mathscr{D}_{-}^{1 / 2} \hat{\omega}=0 .
$$

In components $\hat{\omega}$ and $\mathscr{D}_{-}^{1 / 2} \hat{\omega}$ can be expanded as

$$
\begin{aligned}
\hat{\omega} & =\hat{\omega}_{+}+\theta \hat{\omega}_{z}, \\
\mathscr{D}_{-}^{1 / 2} \hat{\omega} & =\bar{\theta}\left(\partial_{\bar{z}} \hat{\omega}_{+}+\frac{1}{2} \chi_{\bar{z}}^{+} \hat{\omega}_{z}\right)+\bar{\theta} \theta\left(\partial_{\bar{z}} \hat{\omega}_{z}+\frac{1}{2} \partial_{z}\left(\chi_{\bar{z}}^{+} \hat{\omega}_{+}\right)\right),
\end{aligned}
$$

and the equation for superholomorphicity is equivalent to the system

$$
\partial_{\bar{z}} \hat{\omega}_{+}+\frac{1}{2} \chi_{\bar{z}}^{+} \hat{\omega}_{z}=0, \quad \partial_{\bar{z}} \hat{\omega}_{z}+\frac{1}{2} \partial_{z}\left(\chi_{\bar{z}}^{+} \hat{\omega}_{+}\right)=0 .
$$

It is easy to see that this system admits $h$ independent solutions, so that the space of super abelian differentials for even spin structures is $h$-dimensional. It is an important issue to produce a basis with the correct normalizations. We begin by noting that solutions of (5.7) can be generated perturbatively from abelian differentials. In fact since the Dirac operator is invertible for generic even spin structures, we can solve the first equation for $\hat{\omega}_{+}$and substitute in the second equation

$$
\begin{aligned}
& \left(\hat{\omega}_{z}\right)_{I}(z)=\omega_{I}(z)+\frac{1}{16 \pi^{2}} \int d^{2} w d^{2} y \partial_{z} \ln E(z, w) \partial_{w}\left(\chi_{\bar{w}}^{+} S_{\delta}(w, y) \chi_{\bar{y}}^{+}\left(\hat{\omega}_{y}\right)_{I}\right), \\
& \left(\hat{\omega}_{+}\right)_{I}(z)=-\frac{1}{4 \pi} \int d^{2} w S_{\delta}(z, w) \chi_{\bar{w}}^{+}\left(\hat{\omega}_{w}\right)_{I}(w) .
\end{aligned}
$$

These formulas actually determine easily the full solution since they can be iterated to give a series in $\chi_{\bar{z}}^{+}$. In practice $\chi_{\bar{z}}^{+}$will depend on only $2 h-2$ Grassmann variables which parametrize the odd supermoduli of the super Riemann surface, so that the series will terminate after $h$ terms. It should be noted however that the 
integrals in (5.8) should be carried out over the cut Riemann surface, due to the multiple-valuedness of $\partial_{z} \ln E(z, w)$. Thus the super abelian differentials in (5.8) depend on the choice of cuts within the homology classes. A more convenient basis of super abelian differentials which depends only on the homology classes and not on the curves themselves is given instead by

$$
\begin{aligned}
& \left(\hat{\omega}_{z}\right)_{I}(z)=\omega_{I}(z)-\frac{1}{16 \pi^{2}} \int d^{2} w d^{2} y \partial_{z} \partial_{w} \ln E(z, w) \chi_{\bar{w}}^{+} S_{\delta}(w, y) \chi_{\bar{y}}^{+}\left(\hat{\omega}_{y}\right)_{I}, \\
& \left(\hat{\omega}_{+}\right)_{I}(z)=-\frac{1}{4 \pi} \int d^{2} w S_{\delta}(z, w) \chi_{\bar{w}}^{+}\left(\hat{\omega}_{w}\right)_{I}(w) .
\end{aligned}
$$

The integrals in (5.9) are only conditionally convergent due to the double pole in $\partial_{z} \partial_{w} \ln E(z, w)$. If we denote the original solutions found in (5.8) by $\hat{\omega}_{I}^{N}$ to distinguish them from the $\hat{\omega}_{I}$ of (5.9), their difference can be evaluated by an integration by parts using the monodromy of the prime form. We obtain in this way

$$
\begin{aligned}
\left(\hat{\omega}_{z}^{N}\right)_{I}(z)= & \omega_{I}(z)+\frac{1}{4 \pi} \int d^{2} w \partial_{z} \partial_{w} \ln E(z, w) \chi_{\bar{w}}^{+}\left(\hat{\omega}_{+}^{N}\right)_{I}(w) \\
& -\frac{1}{2} \sum_{K=1}^{h} \omega_{K}(z) \oint_{A_{K}} d \bar{w} \chi_{\bar{w}}^{+}\left(\hat{\omega}_{+}^{N}\right)(w) .
\end{aligned}
$$

Since the relation between the two bases is linear it follows that

$$
\left(\hat{\omega}_{z}^{N}\right)_{I}(z)=\left(\hat{\omega}_{z}\right)_{I}(z)-\frac{1}{2} \sum_{K=1}^{h}\left(\hat{\omega}_{z}\right)_{K}(z) \oint_{A_{K}} d \bar{w} \chi_{\bar{w}}^{+}\left(\hat{\omega}_{+}^{N}\right)_{I}(w) .
$$

Thus the relative normalization of the two bases of solutions is described by the $h \times h$ matrix $M_{I K}$,

$$
\begin{aligned}
M_{I K} & =\delta_{I K}-\frac{1}{2} \oint_{A_{K}} d \bar{w} \chi_{\bar{w}}^{+}\left(\hat{\omega}_{+}^{N}\right)(w), \\
\left(\hat{\omega}_{z}\right)_{I}(z) & =\sum_{K=1}^{h} M_{I K}^{-1}\left(\hat{\omega}_{z}^{N}\right)_{K}(z) .
\end{aligned}
$$

We now require a notion of line integrals in supergeometry. Let $\hat{\omega}$ be a super holomorphic $1 / 2$-form. Then over simply connected domains the line integral

$$
\int_{\mathbf{P}}^{z} \hat{\omega} \equiv \int_{P}^{z}\left(d z \hat{\omega}_{z}-\frac{1}{2} d \bar{z} \chi_{\bar{z}}^{+} \hat{\omega}_{+}\right)+\theta_{z} \hat{\omega}_{+}(z)-\theta_{P} \hat{\omega}_{+}(P)
$$

is independent of the choice of paths. In superfield language the difference between two paths is the integral over the region they enclose of $d^{2} z d \theta d \bar{\theta} \mathscr{D}_{-}^{1 / 2} \hat{\omega}$ which is zero since $\hat{\omega}$ is super holomorphic. The resulting function of $\mathbf{z}$ is thus welldefined and satisfies

$$
\mathscr{D}+\int_{\mathbf{P}}^{\mathbf{z}} \hat{\omega}=\hat{\omega}(\mathbf{z}), \quad \mathscr{D}-\int_{\mathbf{P}}^{\mathbf{z}} \hat{\omega}=0 .
$$


The super line integrals provide us with a generalization of the usual periods of abelian differentials, which will again depend only on the basis of homology cycles and not on the choice of curves within a given class. More precisely we may define the period of the super abelian differential $\hat{\omega}$ around a closed curve $C$ as

$$
\oint_{C} \hat{\omega}=\oint_{C}\left(d z \hat{\omega}_{z}-\frac{1}{2} d \bar{z} \chi_{\bar{z}}^{+} \hat{\omega}_{+}\right)
$$

which depends only on the class of $C$.

Returning to the two bases of super abelian differentials of (5.8) and (5.9), we evaluate their periods. Due to the conditional convergence of the integrals defining $\hat{\omega}_{I}$, we cannot interchange the $d z$ integrals around a given cycle and the $d^{2} w$ integrals in (5.9). We shall provide shortly a rule for correction terms that allow such interchanges. At this point it may be instructive to start rather from the periods of the cut-dependent basis $\hat{\omega}_{I}$ which are easier since the integrals there are absolutely convergent. For the $A_{I}$ cycles the results are

$$
\begin{aligned}
\oint_{A_{I}} d z\left(\hat{\omega}_{z}^{N}\right)_{J}-\frac{1}{2} d \bar{z} \chi_{\bar{z}}^{+}\left(\hat{\omega}_{+}^{N}\right)_{J}= & \delta_{I J}-\frac{1}{2} \oint_{A_{I}} \chi_{\bar{z}}^{+}\left(\hat{\omega}_{+}^{N}\right)_{J} \\
& -\frac{1}{4 \pi} \int d^{2} w \oint_{A_{I}} d z \partial_{z} \ln E(z, w) \\
& \times \partial_{w}\left(\chi_{\bar{w}}^{+}\left(\hat{\omega}_{+}^{N}\right)_{J}(w)\right) \\
= & \delta_{I J}-\frac{1}{2} \oint_{A_{I}} d \bar{z} \chi_{\bar{z}}\left(\hat{\omega}_{+}^{N}\right)_{J},
\end{aligned}
$$

where we have used (2.10). It is easy to convert (5.16) into a statement about $\hat{\omega}_{I}$ in view of (5.12),

$$
\oint_{A_{I}} \hat{\omega}_{J}=\delta_{I J} .
$$

Next the periods of $\hat{\omega}^{N}$ around the $B_{I}$ cycles are given by

$$
\begin{aligned}
\oint_{B_{J}} d z\left(\hat{\omega}_{z}^{N}\right)_{I}-\frac{1}{2} d \bar{z} \chi_{\bar{z}}\left(\hat{\omega}_{+}^{N}\right)_{I}= & \Omega_{I J}-\frac{1}{2} \oint_{B_{J}} d \bar{z} \chi_{\bar{z}}\left(\hat{\omega}_{+}^{N}\right)_{I} \\
& -\frac{1}{4 \pi} \int d^{2} w \oint_{B_{J}} d z \partial_{z} \ln E(z, w) \\
& \times \partial_{w}\left(\chi_{\bar{w}}^{+}\left(\hat{\omega}_{+}^{N}\right)_{I}(w)\right) \\
= & \Omega_{I J}-\frac{1}{2} \oint_{B_{J}} d \bar{z} \chi_{\bar{z}}\left(\hat{\omega}_{+}^{N}\right)_{I} \\
& -\frac{1}{4 \pi} \int d^{2} w\left(-i \pi \Omega_{J J}+2 \pi i \int_{z}^{w} \omega_{J}\right) \\
& \times \partial_{w}\left(\chi_{\bar{w}}^{+}\left(\hat{\omega}_{+}^{N}\right)_{I}(w)\right),
\end{aligned}
$$


again using (2.10). Since $\chi_{\bar{w}}^{+} \hat{\omega}_{+}^{N}$ is single-valued, the term $\Omega_{J J}$ does not contribute. An integration by parts with respect to $w$ produces

$$
\begin{aligned}
\oint_{B_{J}} d z\left(\hat{\omega}_{z}^{N}\right)_{I}-\frac{1}{2} d \bar{z} \chi_{\bar{z}}^{+}\left(\hat{\omega}_{+}^{N}\right)_{I}= & \Omega_{I J}-\frac{1}{2} \oint_{B_{J}} d \bar{z} \chi_{\bar{z}}^{+}\left(\hat{\omega}_{+}^{N}\right)_{I} \\
& +\frac{i}{2} \int d^{2} w \omega_{J}(w) \chi_{\bar{w}}^{+}\left(\hat{\omega}_{+}^{N}\right)_{I}(w) \\
& -\frac{1}{2} \oint_{A_{K}} d \bar{w} \chi_{\bar{w}}^{+}\left(\hat{\omega}_{+}^{N}\right)_{I} \oint_{B_{K}} \omega_{J} \\
& +\frac{1}{2} \oint_{A_{K}} \omega_{J} \oint_{B_{K}} d \bar{w} \chi_{\bar{w}}^{+}\left(\hat{\omega}_{+}^{N}\right)_{I} \\
= & \Omega_{I J}+\frac{i}{2} \int d^{2} w \omega_{J}(w) \chi_{\bar{w}}^{+}\left(\hat{\omega}_{+}^{N}\right)_{I}(w) \\
& -\frac{1}{2} \Omega_{K J} \oint_{A_{K}} d \bar{w} \chi_{\bar{w}}^{+}\left(\hat{\omega}_{+}^{N}\right)_{I}(w) .
\end{aligned}
$$

In terms of $\hat{\omega}_{I}$ this is equivalent to

$$
\oint_{B_{J}} \hat{\omega}_{I}=\Omega_{I J}+\frac{i}{2} \int d^{2} w \omega_{J}(w) \chi_{\bar{w}}\left(\hat{\omega}_{+}\right)_{I}(w) .
$$

In practice we see then that the results for line integrals of the super abelian differentials $\hat{\omega}_{I}$ are the same as if we had treated the integrals in (5.9) as absolutely convergent, and ignored the correction terms $\chi_{\bar{z}}^{+} \hat{\omega}$ in the contour integrals. More precisely the formulas (5.17) and (5.20) for the periods can also be derived from the following rule for interchanging the order of integration in the presence of conditionally convergent integrals:

$\frac{1}{2 \pi} \int_{P}^{z} \int d^{2} w \partial_{z} \partial_{w} \ln E(z, w) \phi_{\bar{w}}-\int_{P}^{z} d \bar{z} \phi_{\bar{z}}=\frac{1}{2 \pi} \int d^{2} w \int_{P}^{z} \partial_{z} \partial_{w} \ln E(z, w) \phi_{\bar{w}}$.

Indeed both sides are path independent, the left-hand side because the integrand is a closed form, and the right-hand side because $\partial_{z} \partial_{w} \ln E(z, w)$ has a double pole. Furthermore they have the same $\partial_{z}$ and $\partial_{\bar{z}}$ derivatives. We note that this argument holds as long as $\phi$ is smooth, and does not require specific information about the boundary values of $\phi$.

The relation (5.17) exhibits the $\hat{\omega}_{I}$ as the basis of super abelian differentials which are cut-independent and dual to the homology cycles $A_{I}$. We may thus define the right-hand side of (5.20) as the super period matrix. It can also be written as

$$
\hat{\Omega}_{I J}=\Omega_{I J}-\frac{i}{8 \pi} \int d^{2} y d^{2} w \omega_{I}(w) \chi_{\bar{w}}^{+} S_{\delta}(w, y) \chi_{\bar{y}}^{+}\left(\hat{\omega}_{y}\right)_{J}
$$

From the above expressions it is not evident that $\hat{\Omega}$ is symmetric. This can only be seen by using the Green's function $\hat{S}_{\delta}(z, w)$ for the generalized Dirac operator $\hat{\partial}_{\bar{z}}$ of (4.21) which is defined by

$$
\hat{\partial}_{\bar{z}} \hat{S}_{\delta}(z, w)=2 \pi \delta(z-w) .
$$


This is equivalent to the integral equation

$$
\hat{S}_{\delta}(z, w)=S_{\delta}(z, w)-\frac{1}{2 \pi} \int d^{2} u d^{2} v S_{\delta}(z, u) K(u, v) \hat{S}_{\delta}(v, w),
$$

which again gives immediately the full solution by iteration. A useful identity linking $\hat{S}_{\delta}, S_{\delta}, \hat{\omega}_{I}$, and $\omega_{I}$ is

$$
\int d^{2} y S_{\delta}(w, y) \chi_{\bar{y}}^{+}\left(\hat{\omega}_{y}\right)_{J}=\int d^{2} y \hat{S}_{\delta}(w, y) \chi_{\bar{y}}^{+} \omega_{J}(y) .
$$

This is a consequence of (5.9), of iteration procedures, and the fact that

$$
\chi_{\bar{z}}^{+}\left(\hat{\omega}_{z}\right)_{J}=\chi_{\bar{z}}^{+} \omega_{J}(z)+\frac{1}{2 \pi} \int d^{2} w d^{2} y K(z, w) S_{\delta}(w, y) \chi_{\bar{z}}^{+}\left(\hat{\omega}_{y}\right)_{J} .
$$

Thus the super-period matrix can also be written in the manifestly symmetric form

$$
\hat{\Omega}_{I J}=\Omega_{I J}-\frac{i}{8 \pi} \int d^{2} w d^{2} y \omega_{J}(w) \chi_{\bar{w}}^{+} \hat{S}_{\delta}(w, y) \chi_{\bar{y}}^{+} \omega_{I}(y) .
$$

A final crucial superconformal invariant is a super analogue $\mathscr{E}_{\delta}(\mathbf{z}, \mathbf{w})$ of the prime form. A guiding principle for the definition of this "super prime form" is that it should give the full (i.e. in presence of a non-vanishing gravitino $\chi_{\bar{z}}^{+}$) twopoint function of the effective chiral field $X_{+}$

$$
\mathscr{G}_{+}(\mathbf{z}, \mathbf{w})=\left\langle X_{+}(\mathbf{z}) X_{+}(\mathbf{w})\right\rangle_{x_{+}}=-\ln \mathscr{E}_{\delta}(\mathbf{z}, \mathbf{w}),
$$

and thus lead to a simple expression for exponential insertions

$$
\frac{\left\langle\exp \left[-\frac{1}{4 \pi} \int d^{2} z \chi_{\bar{z}}^{+} \psi_{+}^{\mu} \partial_{z} x_{+}^{\mu}+i \sum_{i=1}^{n} k_{i}^{\mu} X_{+}\left(\mathbf{z}_{i}\right)\right]\right\rangle_{x, \psi}}{\left\langle\exp \left[-\frac{1}{4 \pi} \int d^{2} z \chi_{\bar{z}}^{+} \psi_{+}^{\mu} \partial_{z} x_{+}^{\mu}\right]\right\rangle_{x, \psi}}=\prod_{i<j} \mathscr{E}_{\delta}\left(\mathbf{z}_{i}, \mathbf{z}_{j}\right)^{k_{i} k_{j}} .
$$

These are evidently the super versions of the fundamental properties (4.27a) and (4.27b) of the usual prime form. Performing $x_{+}$and $\psi_{+}$contractions according to (4.27) and (5.1), we obtain an explicit expression in components

$$
\begin{aligned}
-\ln \mathscr{E}_{\delta}(\mathbf{z}, \mathbf{w})= & -\ln E(z, w)+\theta_{z} \theta_{w} \hat{S}_{\delta}(z, w) \\
& +\frac{1}{4 \pi} \theta_{z} \int d^{2} y \chi_{\bar{y}}^{+} \partial_{y} \ln E(y, w) \hat{S}_{\delta}(z, y) \\
& +\frac{1}{4 \pi} \theta_{w} \int d^{2} y \chi_{\bar{y}}^{+} \partial_{y} \ln E(y, z) \hat{S}_{\delta}(w, y) \\
& +\frac{1}{16 \pi^{2}} \int d^{2} x d^{2} y \chi_{\bar{x}}^{+} \partial_{x} \ln E(x, z) \chi_{\bar{y}}^{+} \partial_{y} \ln E(y, w) \hat{S}_{\delta}(x, y) \\
& +f(\mathbf{z})+g(\mathbf{w}) .
\end{aligned}
$$

The functions $f(\mathbf{z})$ and $g(\mathbf{w})$ are at this point arbitrary. They are not determined by (5.29) alone, since their contributions cancel out due to overall momentum conservation $\sum k_{i}=0$. Also the functions $\partial_{y} \ln E(y, w)$ and $\partial_{y} \ln E(y, z)$ have 
monodromies, so the integrals in (5.30) depend on the choice of fundamental domain over which we are carrying out the integration. This ambiguity is again of the form $f(\mathbf{z})+g(\mathbf{w})$, and we can view it as absorbed in the earlier ambiguity. We observe that the chiral amplitudes themselves are always well defined.

We shall now show that the functions $f(\mathbf{z})$ and $g(\mathbf{w})$ can actually be fixed by additional natural conditions on the monodromy of the super prime form. First, requiring that $\mathscr{E}_{\delta}(\mathbf{z}, \mathbf{w})$ be antisymmetric implies that $f=g$. Next we note that

$$
\partial_{+}^{\mathbf{z}} \partial_{+}^{\mathbf{w}} \ln \mathscr{E}_{\delta}(\mathbf{z}, \mathbf{w})
$$

is well-defined and has no monodromy. Its periods around the basis homology cycles are unambiguous and can be evaluated to be

$$
\begin{aligned}
& \oint_{A_{I}} \partial_{+}^{\mathbf{z}} \partial_{+}^{w} \ln \mathscr{E}_{\delta}(\mathbf{z}, \mathbf{w})=0, \\
& \oint_{B_{I}} \partial_{+}^{\mathbf{z}} \partial_{+}^{w} \ln \mathscr{E}_{\delta}(\mathbf{z}, \mathbf{w})=2 \pi i \hat{\omega}_{I}(\mathbf{w}) .
\end{aligned}
$$

Integrating $\partial_{+}^{\mathbf{z}} \partial_{+}^{\mathbf{w}} \ln \mathscr{E}_{\delta}(\mathbf{z}, \mathbf{w})$ with respect to $\mathbf{z}$ shows that the monodromy of $\partial_{+}^{w} \ln \mathscr{E}_{\delta}(\mathbf{z}, \mathbf{w})$ as a function of $\mathbf{z}$ must be a function of $\mathbf{w}$ alone. In view of (5.31) we must have

$$
\begin{aligned}
& \partial_{+}^{w} \ln \mathscr{E}_{\delta}\left(\mathbf{z}+A_{K}, \mathbf{w}\right)=\partial_{+}^{w} \ln \mathscr{E}_{\delta}(\mathbf{z}, \mathbf{w}), \\
& \partial_{+}^{\mathrm{w}} \ln \mathscr{E}_{\delta}\left(\mathbf{z}+B_{K}, \mathbf{w}\right)=\partial_{+}^{\mathrm{w}} \ln \mathscr{E}_{\delta}(\mathbf{z}, \mathbf{w})+2 \pi i \hat{\omega}_{K}(\mathbf{w}) .
\end{aligned}
$$

This monodromy as $z$ is transported around is consistent with (5.30) for any function $f(\mathbf{z})$. If we impose the condition that the super prime form be invariant as both $z$ and $w$ are transported around the same cycle,

$$
\mathscr{E}_{\delta}(\mathbf{z}+C, \mathbf{w}+C)=\mathscr{E}_{\delta}(\mathbf{z}, \mathbf{w}),
$$

we obtain from (5.32) the following monodromy as $w$ is transported around nontrivial cycles

$$
\begin{aligned}
& \partial_{+}^{w} \ln \mathscr{E}_{\delta}\left(\mathbf{z}, \mathbf{w}+A_{K}\right)=\partial_{+}^{w} \ln \mathscr{E}_{\delta}(\mathbf{z}, \mathbf{w}), \\
& \partial_{+}^{w} \ln \mathscr{E}_{\delta}\left(\mathbf{z}, \mathbf{w}+B_{K}\right)=\partial_{+}^{w} \ln \mathscr{E}_{\delta}(\mathbf{z}, \mathbf{w})-2 \pi i \hat{\omega}_{K}(\mathbf{w}),
\end{aligned}
$$

which is incidentally the natural extension of the corresponding property for the usual prime form. On the other hand we can use (5.30) to evaluate the monodromy of $\partial_{+}^{w} \ln \mathscr{E}(\mathbf{z}, \mathbf{w})$ in terms of $f(\mathbf{z})$. In this way we arrive at the equation

$$
2 \pi i \theta_{w} \omega_{K}(w)+\left(\partial_{+} f\left(\mathbf{w}+B_{K}\right)-\partial_{+} f(\mathbf{w})\right)=2 \pi i \hat{\omega}_{K}(w) .
$$

More explicitly

$$
\begin{aligned}
f\left(\mathbf{w}+B_{K}\right)= & f(\mathbf{w})+\frac{i}{2} \theta_{w} \int d^{2} y \chi_{\bar{y}}^{+} \omega_{K}(y) \hat{S}_{\delta}(w, y) \\
& +\frac{i}{8 \pi} \int d^{2} x d^{2} y \chi_{\bar{x}}^{+} \omega_{K}(x) \chi_{\bar{y}}^{+} \partial_{y} \ln E(y, w) \hat{S}_{\delta}(x, y) .
\end{aligned}
$$


This determines $f(\mathbf{w})$ up to an additive constant

$$
\begin{aligned}
f(\mathbf{w})= & \frac{1}{4 \pi} \theta_{w} \int d^{2} y \chi_{\bar{y}}^{+} \partial_{y} \ln E(y, w) \hat{S}_{\delta}(w, y) \\
& +\frac{1}{32 \pi^{2}} \int d^{2} x d^{2} y \chi_{\bar{x}}^{+} \partial_{x} \ln E(x, w) \chi_{\bar{y}}^{+} \partial_{y} \ln E(y, w) \hat{S}_{\delta}(x, y) .
\end{aligned}
$$

Combining this expression with (5.30) we get

$$
\begin{aligned}
-\ln \mathscr{E}_{\delta}(\mathbf{z}, \mathbf{w})= & -\ln E(z, w)+\theta_{z} \theta_{w} \hat{S}_{\delta}(z, w) \\
& +\frac{1}{4 \pi} \theta_{z} \int d^{2} y \chi_{\bar{y}}^{+} \partial_{y} \ln \frac{E(y, w)}{E(y, z)} \hat{S}_{\delta}(z, y) \\
& +\frac{1}{4 \pi} \theta_{w} \int d^{2} y \chi_{\bar{y}}^{+} \partial_{y} \ln \frac{E(y, z)}{E(y, w)} \hat{S}_{\delta}(w, y) \\
& +\frac{1}{32 \pi^{2}} \int d^{2} x d^{2} y \chi_{\bar{x}}^{+} \partial_{x} \ln \frac{E(x, z)}{E(x, w)} \hat{S}_{\delta}(x, y) \chi_{\bar{y}}^{+} \partial_{y} \ln \frac{E(y, w)}{E(y, z)}
\end{aligned}
$$

Remarkably the expression (5.37) is now well-defined on the Riemann surface, and the super prime form for even spin structures is completely unambiguous.

The super prime form shares many properties with the standard prime form; in particular

$$
\left\{\begin{array}{r}
\mathscr{E}_{\delta}(\mathbf{z}, \mathbf{w})=0 \\
\mathscr{D}_{+}^{\mathbf{z}} \mathscr{E}_{\delta}(\mathbf{z}, \mathbf{w})=0
\end{array}\right\} \Leftrightarrow\left\{\begin{array}{c}
z=w \\
\theta_{z}=\theta_{w}
\end{array}\right\}
$$

(ii) $\mathscr{E}_{\delta}(\mathbf{z}, \mathbf{w})$ is a $(-1 / 2,0) \times(-1 / 2,0)$ form in $\mathbf{z}$ and $\mathbf{w}$,

(iii) $\mathscr{E}_{\delta}(\mathbf{z}, \mathbf{w})$ is superholomorphic in all its arguments,

$$
\begin{aligned}
& \mathscr{E}_{\delta}\left(\mathbf{z}+A_{I}, \mathbf{w}\right)=\mathscr{E}_{\delta}(\mathbf{z}, \mathbf{w}), \\
& \mathscr{E}_{\delta}\left(\mathbf{z}+B_{I}, \mathbf{w}\right)=\mathscr{E}_{\delta}(\mathbf{z}, \mathbf{w}) \exp \left(-i \pi \hat{\Omega}_{I I}+2 \pi i \int_{\mathbf{z}}^{\mathbf{w}} \hat{\omega}_{I}\right) .
\end{aligned}
$$

The properties (i), (iii), (iv) are direct consequences of the component expression (5.37), while (ii) is a consequence of the local supersymmetry covariance of the expression (5.29). However, since $\mathscr{E}_{\delta}$ involves the Szego kernel with spin structure $\delta$, it depends on a spin structure as opposed to the ordinary prime form which does not.

We can now produce a more intrinsic version of the chiral amplitudes $\mathscr{F}_{\delta}$. First, the source-independent part of $\mathscr{F}_{\delta}^{m}$ is a power of the chiral scalar superfield determinant:

$$
\frac{s \operatorname{det}^{\prime} \mathscr{D}+\mathscr{D}_{-}^{(0)}}{\operatorname{det} \operatorname{Im} \hat{\Omega} \int d^{2} \mathbf{z} E}=\left|Z_{\mathscr{D}, \delta}\left(\hat{\Omega}, \chi_{\bar{z}}^{+}\right)\right|^{4} \exp \left(-2 S_{s L}\right),
$$

and we have the relation

$$
Z_{\mathscr{D}, \delta}\left(\hat{\Omega}, \chi_{\bar{z}}^{+}\right)^{-1}=Z_{\Delta}(\Omega)^{-1} Z_{\delta}(\Omega)\left\langle\exp \left[-\frac{1}{4 \pi} \int d^{2} z \chi_{\bar{z}}^{+} \psi_{+}^{\mu} \partial_{z} x_{+}^{\mu}\right]\right\rangle_{x, \psi} .
$$


The action $S_{s L}$ is the super Liouville action which expresses the super conformal anomaly

$$
S_{s L}\left(\ln g_{z \bar{z}}, \chi_{z}^{+}\right)=\frac{1}{48 \pi} \int d^{2} z\left(\partial_{z} \ln g_{z \bar{z}} \partial_{\bar{z}} \ln g_{z \bar{z}}+\chi_{z}^{+} \partial_{\bar{z}} \chi_{z}^{+}+\chi_{\bar{z}}^{-} \partial_{z} \chi_{\bar{z}}^{-}\right) .
$$

Since we have dropped the $\gamma$-traces of the world-sheet gravitino, it reduces to the Liouville action. The supersymmetry anomaly of the chiral super determinant is given by

$$
\delta \ln Z_{\mathscr{D}, \delta}=\frac{1}{8 \pi} \int d^{2} z \chi_{\bar{z}}^{+} \partial_{z}^{2} \zeta^{+}(z) .
$$

Altogether (5.4), (5.29), and (5.40) combine to give a manifestly supersymmetric formula for $\mathscr{F}_{\delta}^{m}$, up to the anomaly of (5.42),

$$
\begin{aligned}
\mathscr{F}_{\delta}^{m}= & Z_{\mathscr{D}, \delta}\left(\hat{\Omega}, \chi_{\bar{z}}^{+}\right)^{-10} \exp \left[i \pi p_{I}^{\mu} \hat{\Omega}_{I J} p_{J}^{\mu}+2 \pi p_{I}^{\mu} \sum_{i=1}^{n}\left(i k_{i}^{\mu}+\zeta_{i}^{\mu} \partial_{+}^{i}\right) \int_{P}^{\mathbf{z}_{i}} \hat{\omega}_{I}\right] \\
& \times\left\langle\exp \left[\sum_{i=1}^{n}\left(i k_{i}^{\mu} X_{+}^{\mu}\left(\mathbf{z}_{i}\right)+\zeta_{i}^{\mu} \partial_{+} X_{+}^{\mu}\left(\mathbf{z}_{i}\right)\right)\right]\right\rangle_{X_{+}} .
\end{aligned}
$$

In the above formula the indefinite line integrals of the super abelian differentials are taken in the sense of (5.13) and in particular path-independent. One can check that it coincides with the component expression of (5.4) by arguments similar to those used in the evaluation of periods (5.17) and (5.20), where the contributions of $\chi_{\bar{z}}^{+} \psi+$ cancel those of the double pole in the definition of $\hat{\omega}_{I}$. The contractions in $X_{+}$are to be carried out with the super Green's function $-\ln \mathscr{E}_{\delta}(\mathbf{z}, \mathbf{w})$ of $(5.28)$. In terms of the super prime form they can be rewritten as

$$
\begin{aligned}
& \left\langle\exp \left[\sum_{i=1}^{n}\left(i k_{i}^{\mu} X_{+}^{\mu}\left(\mathbf{z}_{i}\right)+\zeta_{i}^{\mu} \partial_{+} X_{+}^{\mu}\left(\mathbf{z}_{i}\right)\right)\right]\right\rangle_{X_{+}} \\
& \quad=\exp \left[\sum_{i, j=1}^{n}\left(\frac{1}{2} k_{i} k_{j} \ln \mathscr{E}_{\delta}\left(\mathbf{z}_{i}, \mathbf{z}_{j}\right)+\frac{1}{2} \zeta_{i} \zeta_{j} \partial_{+}^{i} \partial_{+}^{j} \ln \mathscr{E}_{\delta}\left(\mathbf{z}_{i}, \mathbf{z}_{j}\right)-i k_{i} \zeta_{j} \partial_{+}^{j} \ln \mathscr{E}_{\delta}\left(\mathbf{z}_{i}, \mathbf{z}_{j}\right)\right)\right] .
\end{aligned}
$$

These are the chirally split, manifestly super-reparametrization invariant expressions for the scalar superfields correlation functions that we have been looking for. Translation invariance in $X_{+}^{\mu}$ guarantees that the full correlation functions are well-defined on the super Riemann surface.

A last equivalent form for chiral amplitudes is obtained by introducing the internal loop momenta of the effective scalar superfields,

$\mathscr{\mathscr { F }}_{\delta}^{m}=Z_{\mathscr{Q}, \delta}\left(\hat{\Omega}, \chi_{\bar{z}}^{+}\right)^{-10}\left\langle\exp \left[i p_{I}^{\mu} \oint_{B_{I}} d \mathbf{z} \partial_{+} X_{+}^{\mu}+\sum_{i=1}^{n} i k_{i}^{\mu} X_{+}^{\mu}\left(\mathbf{z}_{i}\right)+\zeta_{i}^{\mu} \partial_{+} X_{+}^{\mu}\left(\mathbf{z}_{i}\right)\right]\right\rangle_{X_{+}}$.

The chiral amplitude $\mathscr{F}_{\delta}^{m}$ is a form of weight $(1 / 2,0)$ in each of the insertion points $\mathbf{z}_{i}$, with monodromy only around $B_{K}$ cycles:

$\mathscr{F}_{\delta}^{m}\left(\mathbf{z}_{i}+\delta_{i j} B_{K}, \zeta_{i}^{\mu}, k_{i}^{\mu} ; \hat{\Omega}, \chi_{\bar{z}}^{+} ; p_{I}^{\mu}\right)=\mathscr{F}_{\delta}^{m}\left(\mathbf{z}_{i}, \zeta_{i}^{\mu}, k_{i}^{\mu} ; \hat{\Omega}, \chi_{\bar{z}}^{+} ; p_{I}^{\mu}+\delta_{I K} k_{j}^{\mu}\right)$. 
In view of translation invariance of the integration domain of the internal momenta $p_{I}^{\mu}$, the monodromy is cancelled out in the full integrated amplitude $\mathscr{A}_{\delta}$, which is then a regular form.

\section{Superabelian Differentials, Super-Period Matrix, and Chiral Amplitudes for Odd Spin Structure}

We come now to the more complicated case of generic super Riemann surfaces with odd spin structures. The additional difficulties arise from the zero mode of the Dirac operator on rank $1 / 2$ tensors, which has been written down explicitly in (2.8). Thus the field $\psi_{+}$should be decomposed as

$$
\psi_{+}^{\mu}(z)=\psi_{+}^{\prime \mu}(z)+\psi_{+}^{0, \mu} \frac{h_{\delta}(z)}{\left\langle h_{\delta} \mid h_{\delta}\right\rangle^{1 / 2}}, \quad\left\langle\psi_{+}^{\prime \mu} \mid h_{\delta}\right\rangle=0,
$$

and the propagator satisfies the modified equation

$$
\begin{gathered}
S_{\delta}^{\prime}(z, w)=-\left\langle\psi_{+}^{\prime}(z) \psi_{+}^{\prime}(w)\right\rangle_{\psi^{\prime}} \\
D_{\bar{z}}^{1 / 2} S_{\delta}^{\prime}(z, w)=2 \pi \delta(z-w)-2 \pi \frac{\bar{h}_{\delta}(z) h_{\delta}(w)}{\left\langle h_{\delta} \mid h_{\delta}\right\rangle} .
\end{gathered}
$$

Due to the last term in (6.2), $S_{\delta}^{\prime}$ cannot be meromorphic in its arguments however. We can construct a meromorphic propagator, at the expense of introducing a pole at an arbitrary point $Q$ on the surface

$$
D_{\bar{z}} S_{\delta}^{\prime \prime}(z, w ; Q)=2 \pi \delta(z-w)-2 \pi \delta(z-Q) \frac{h_{\delta}(w)}{h_{\delta}(Q)} .
$$

In terms of the $\vartheta$-function and the prime form, it can be expressed as

$$
S_{\delta}^{\prime \prime}(z, w ; Q)=\frac{1}{E(z, w)} \frac{\vartheta[\delta](z-w+P-Q)}{\vartheta[\delta](P-Q)} \frac{E(z, P) E(w, Q)}{E(z, Q) E(w, P)},
$$

where we have identified its additional zero $P$. Another possibility is to rely on a meromorphic propagator without any additional pole, but which is defined only on the universal covering of the surface. Such propagators can be parametrized by the choice of an arbitrary generic point $V_{I}$ in the Jacobian, after which it can be characterized by

$$
\begin{aligned}
D_{\bar{z}}^{1 / 2} S_{\delta}(z, w ; V) & =2 \pi \delta(z-w), \\
S_{\delta}(z, w ; V) & =\frac{1}{E(z, w)} \frac{\sum_{I=1}^{h} \partial_{z_{I}} \vartheta[\delta](z-w, \Omega) V_{I}}{\sum_{I=1}^{h} \partial_{z_{I}} \vartheta[\delta](0, \Omega) V_{I}} .
\end{aligned}
$$


The monodromy of this propagator can be described as follows. When $z$ is taken around

- an $A_{I}$ cycle,

$$
S_{\delta}\left(z+A_{K}, w ; V\right)=-\exp \left(-2 \pi i \delta_{K}^{\prime}\right) S_{\delta}(z, w ; V)
$$

- a $B_{I}$ cycle,

$$
S_{\delta}\left(z+B_{K}, w ; V\right)=\exp \left(-2 \pi i \delta_{K}^{\prime \prime}\right)\left[S_{\delta}(z, w ; V)+h_{\delta}(z) \hat{V}_{K} h_{\delta}(w)\right],
$$

where

$$
\hat{V}_{K}=-2 \pi i \frac{V_{K}}{\sum_{I=1}^{h} \partial_{z_{I}} \vartheta[\delta](0, \Omega) V_{I}} .
$$

Upon specializing to the case where $V_{K}$ is a vector of abelian differentials evaluated at a fixed point $Q$

$$
V_{K}=\omega_{K}(Q),
$$

we recover the propagator introduced by Verlinde and Verlinde [24]. The monodromy is then given by

$$
\hat{V}_{K}=-2 \pi i \frac{\omega_{K}(Q)}{h_{\delta}^{2}(Q)} .
$$

Any two of these different propagators are linked by the same relation. For example $S_{\delta}^{\prime}(z, w)$ can be written as

$$
\begin{aligned}
S_{\delta}^{\prime}(z, w)= & S_{\delta}(z, w)-\frac{h_{\delta}(z)}{\left\langle h_{\delta} \mid h_{\delta}\right\rangle} \int d^{2} y \bar{h}_{\delta}(y) S_{\delta}(y, w) \\
& -\frac{h_{\delta}(w)}{\left\langle h_{\delta} \mid h_{\delta}\right\rangle} \int d^{2} y \bar{h}_{\delta}(y) S_{\delta}(z, y) \\
& +\frac{h_{\delta}(z) h_{\delta}(w)}{\left\langle h_{\delta} \mid h_{\delta}\right\rangle^{2}} \int d^{2} y d^{2} u \bar{h}_{\delta}(y) \bar{h}_{\delta}(u) S_{\delta}(y, u)
\end{aligned}
$$

where $S_{\delta}(z, w)$ could be either one of the other two propagators.

We return to the chiral amplitudes $\mathscr{F}_{\delta}^{m}$ of (4.25)-(4.28) in the case of odd spin structure. In principle the $\psi_{+}$contractions occurring there should be carried out with the propagator $S_{\delta}^{\prime}(z, w)$ of $(6.1)$. At first sight it may seem that holomorphicity is lost, but we shall presently show that it will be restored upon integration over the Dirac zero mode.

To see this, we begin by isolating all terms in (4.28) involving the field $\psi_{+}$; they can be recast in a simple form upon introducing a source $j^{\mu}$ :

$$
\begin{aligned}
\mathscr{F}_{\delta}^{\psi} & =\int D \psi_{+}^{\mu} \exp \left[-I_{0}\left(\psi_{+}\right)+\left(j^{\mu} \mid \psi_{+}^{\mu}\right)\right], \\
j^{\mu}(z) & =-\frac{1}{4 \pi} \chi_{\bar{z}}^{+}(z) \partial_{z} x_{+}^{\mu}(z)+\sum_{i=1}^{n}\left(i k_{i}^{\mu} \theta_{i}+\zeta_{i}^{\mu}\right) \delta\left(z-z_{i}\right), \\
\left(j^{\mu} \mid \psi_{+}^{\mu}\right) & =\int j^{\mu}(z) \psi_{+}^{\mu}(z) .
\end{aligned}
$$


We then split the measure according to the decomposition (6.1):

$$
\mathscr{F}_{\delta}^{\psi}=\int D \psi_{+}^{\prime \mu} d \psi_{+}^{0, \mu} \exp \left[-I_{0}\left(\psi_{+}^{\prime}\right)+\left\langle j^{\mu} \mid \psi_{+}^{\prime \mu}\right\rangle+\psi_{+}^{0, \mu} \frac{\left(j^{\mu} \mid h_{\delta}\right)}{\left(h_{\delta} \mid h_{\delta}\right)^{1 / 2}}\right] .
$$

In terms of the propagator $S_{\delta}^{\prime}(z, w)$ this is readily evaluated to be

$$
\mathscr{F}_{\delta}^{w}=Z_{\delta}(\Omega)^{10} \exp \left(-\frac{1}{2} \int d^{2} z d^{2} w j^{\mu}(z) S_{\delta}^{\prime}(z, w) j^{\mu}(w)\right) \varepsilon_{\mu_{1} \ldots \mu_{10}} \prod_{\alpha=1}^{10}\left(j^{\mu_{\alpha}} \mid h_{\delta}\right) .
$$

Here the chiral determinant $Z_{\delta}(\Omega)$ is defined as the holomorphic square root of the determinant of the Laplacian on rank $1 / 2$ spinors, normalized with the inner product of its zero mode

$$
\left|Z_{\delta}(\Omega)\right|^{4}=\frac{\operatorname{det}^{\prime} \Delta_{1 / 2}^{-}}{\left(h_{\delta} \mid h_{\delta}\right)^{2}} e^{-S_{L}(\varrho)}
$$

As pointed out before, we can ignore the anomalies of the Liouville term since they will cancel in the end. Powers of $\left(h_{\delta} \mid h_{\delta}\right)$ in (6.10) arise from the $\psi_{+}^{\prime}$ integration, but also from the zero mode factor in (6.9). It is now clear that $\mathscr{F}_{\delta}^{w}$ is left unchanged under a shift of the form

$$
S_{\delta}^{\prime}(z, w) \rightarrow S_{\delta}^{\prime}(z, w)+h_{\delta}(z) q_{1}(w)+q_{2}(z) h_{\delta}(w)
$$

for arbitrary $q_{1}$ and $q_{2}$. In particular we can replace $S_{\delta}^{\prime}(z, w)$ by either one of the other two propagators and obtain a holomorphic expression for $\mathscr{F}_{\delta}^{\psi}$. We can now assemble the expression obtained for the chiral amplitude in (4.28) with the above result (6.10) for the integration over the chiral fermion field. To simplify the notation, we shall henceforth denote $\psi_{+}^{\prime \mu}$ simply by $\psi_{+}^{\mu}$. Introducing a chiral scalar superfield $X_{+}$as in (5.3) we arrive at

$$
\mathscr{F}_{\delta}^{m}\left(p_{I}^{\mu}\right)=\int d \psi_{+}^{0, \mu} C_{\delta}^{m}\left(p_{I}^{\mu}, \psi_{+}^{\mu, 0}\right),
$$

where

$$
\begin{aligned}
C_{\delta}^{m}\left(p_{I}^{\mu}, \psi_{+}^{\mu, 0}\right)= & Z_{\Delta}^{-10}(\Omega) Z_{\delta}^{10}(\Omega)\left\langle\operatorname { e x p } \left[\psi_{+}^{0, \mu}\left(h_{\delta} \mid j^{\mu}\right)-\frac{1}{4 \pi} \int d^{2} z \chi_{\bar{z}}^{+} \psi_{+}^{\mu} \partial_{z} x_{+}^{\mu}\right.\right. \\
& \left.\left.+i \pi p_{I}^{\mu} \oint_{B_{I}} d z \partial_{z} x_{+}^{\mu}+i \sum_{i=1}^{n} k_{i}^{\mu} X_{+}^{\mu}\left(\mathbf{z}_{i}\right)+\sum_{i=1}^{n} \zeta_{i}^{\mu} \partial_{+} X_{+}^{\mu}\left(\mathbf{z}_{i}\right)\right]\right\rangle
\end{aligned}
$$

and the fermion propagator used to contract $\psi_{+}$may be $S_{\delta}(z, w ; V), S_{\delta}^{\prime}(z, w)$, or $S_{\delta}^{\prime \prime}(z, w ; Q)$.

In summary the chiral amplitude $\mathscr{F}_{\delta}^{m}\left(p_{I}^{\mu}\right)$ can be represented in a number of ways, each with its own advantages and disadvantages. With $S_{\delta}^{\prime}(z, w)$ it is welldefined on the Riemann surface but its holomorphicity is obscured. With both $S_{\delta}(z, w ; V)$ and $S_{\delta}^{\prime \prime}(z, w ; Q)$ it is manifestly holomorphic; however in the first case it appears to be multiple valued while in the second it carries spurious poles. It is likely that the use of $S_{\delta}^{\prime \prime}(z, w)$ will prove particularly convenient when discussing degenerations. In the rest of this paper, we shall instead rely on $S_{\delta}(z, w)=S_{\delta}(z, w$; $V)$, as it will guarantee holomorphicity of intrinsic supergeometric quantities such as super prime forms and super abelian differentials. It will also lead us to 
formulations of correlation functions in terms of conformal blocks, which are of special interest from the point of view of conformal field theory.

\section{Geometry of Super Riemann Surfaces with Odd Spin Structure}

The notions of super Abelian differentials and super-period matrix are more subtle for odd spin structure. To begin with we need a suitable Green's function for the generalized Dirac operator $\hat{\partial}_{\bar{z}}$. There is no canonical choice satisfying, say, the generalized Dirac equation with its zero modes projected out. Rather we shall make use of propagators $\hat{S}_{\delta}(z, w)$ defined through the integral equation

$$
\hat{S}_{\delta}(z, w)=S_{\delta}(z, w)-\frac{1}{2 \pi} \int d^{2} u d^{2} v S_{\delta}(z, u) K(u, v) \hat{S}_{\delta}(v, w) .
$$

Here it is understood that $S_{\delta}(z, w)$ is a generic notation for the various propagators $S_{\delta}(z, w), S_{\delta}^{\prime}(z, w)$, or $S_{\delta}^{\prime \prime}(z, w)$ above, and we shall denote the corresponding $\hat{S}_{\delta}(z, w)$ by $\hat{S}_{\delta}^{\prime}(z, w), \hat{S}_{\delta}(z, w)$, or $\hat{S}_{\delta}^{\prime \prime}(z, w)$ when it is appropriate to make a distinction. They will all satisfy equations of the form

$$
\hat{\partial}_{\bar{z}} \hat{S}_{\delta}=2 \pi \delta(z-w)-M(z, w),
$$

where the correction term $M(z, w)$ is given respectively by

$$
\begin{aligned}
& M(z, w)=0, \\
& M(z, w)=2 \pi \frac{\bar{h}_{\delta}(z) h_{\delta}(w)}{\left\langle h_{\delta} \mid h_{\delta}\right\rangle}-\int d^{2} u d^{2} v \frac{\bar{h}_{\delta}(z) h_{\delta}(w)}{\left\langle h_{\delta} \mid h_{\delta}\right\rangle} K(u, v) \hat{S}_{\delta}^{\prime}(v, w), \\
& M(z, w)=2 \pi \delta(z-Q) \frac{h_{\delta}(w)}{h_{\delta}(Q)}-2 \pi \delta(z-Q) \int \frac{h_{\delta}(u)}{h_{\delta}(Q)} K(u, v) \hat{S}_{\delta}^{\prime \prime}(v, w),
\end{aligned}
$$

in the cases of $\hat{S}_{\delta}(z, w), \hat{S}_{\delta}^{\prime}(z, w)$, and $\hat{S}_{\delta}^{\prime \prime}(z, w)$.

Whereas $\hat{S}^{\prime}$ and $\hat{S}^{\prime \prime}$ are well defined spinors on the Riemann surface, the spinor $\hat{S}_{\delta}(z, w)$ has monodromy. This is of course required by the presence of the zero mode, and the fact that $\hat{S}_{\delta}$ has only one pole. Indeed from (6.6) we readily find that

$$
\begin{aligned}
& \hat{S}_{\delta}\left(z+A_{I}, w\right)=-\exp \left(-2 \pi i \delta_{I}^{\prime}\right) \hat{S}_{\delta}(z, w), \\
& \hat{S}_{\delta}\left(z+B_{I}, w\right)=-\exp \left(-2 \pi i \delta_{I}^{\prime \prime}\right)\left(\hat{S}_{\delta}(z, w)+h_{\delta}(z) \hat{V}_{I}\left(\hat{\omega}_{+}\right)_{0}(w)\right),
\end{aligned}
$$

where

$$
\left(\hat{\omega}_{+}\right)_{0}(w)=h_{\delta}(w)-\frac{1}{2 \pi} \int d^{2} x d^{2} y \hat{S}_{\delta}(w, x) K(x, y) h_{\delta}(y)
$$

The half form $\left(\hat{\omega}_{+}\right)_{0}$ satisfies the homogeneous equation

$$
\hat{\partial}_{\bar{z}}\left(\hat{\omega}_{+}\right)_{0}=0 \text {. }
$$

Furthermore it has no monodromy ${ }^{2}$. In fact the monodromy of $\left(\hat{\omega}_{+}\right)_{0}$ is proportional to

$$
\int d^{2} x d^{2} y\left(\hat{\omega}_{+}\right)_{0}(x) K(x, y) h_{\delta}(y)=-h_{\delta} K\left(1-\frac{1}{2 \pi} \hat{S}_{\delta} K\right) h_{\delta},
$$

which vanishes due to the antisymmetry of $K$ and $\hat{S}_{\delta}$.

\footnotetext{
${ }^{2}$ We regard as trivial the phases associated with the choice of spin structure
} 
A few remarks about allowed monodromies are in order here. Since $\hat{\partial}_{\bar{z}} \hat{S}_{\delta}=2 \pi \delta(z-w)$, we would naively expect that the monodromy of $\hat{S}_{\delta}$ should satisfy the corresponding homogeneous equation. However it should be kept in mind that $\hat{\partial}_{\bar{z}}$ is actually a non-local operator which depends on the choice of fundamental domain for the surface. Thus the monodromy of $\hat{S}_{\delta}$ satisfies only a modified version of the homogeneous equation which takes into account the variation of domains. This is why (6.16) is correct, despite the fact that $\hat{\delta}_{\bar{z}} h_{\delta} \neq 0$. To get constraints on allowed monodromies, we can exploit the fact that $\left(\hat{\omega}_{+}\right)_{0}$ is a solution of the homogeneous equation. If the monodromy of $\hat{S}_{\delta}$ is of the form

$$
\begin{aligned}
& \hat{S}_{\delta}\left(z+A_{K}, w\right)=-\exp \left(-2 \pi i \delta_{K}^{\prime}\right) \hat{S}_{\delta}(z, w), \\
& \hat{S}_{\delta}\left(z+B_{K}, w\right)=-\exp \left(-2 \pi i \delta_{K}^{\prime \prime}\right)\left(\hat{S}_{\delta}(z, w)+\Sigma_{K}(z, w)\right),
\end{aligned}
$$

then as a consequence of the antisymmetry of $\hat{S}_{\delta}$

$$
\hat{\partial}_{\bar{w}} \Sigma_{K}(z, w)=0 .
$$

Also the equation $\hat{\partial}_{\bar{z}} \hat{S}_{\delta}=2 \pi \delta$ implies

$$
\int d^{2} z\left(\hat{\omega}_{+}\right)_{0}(z) \hat{\partial}_{\bar{z}} \hat{S}_{\delta}(z, w)=2 \pi\left(\hat{\omega}_{+}\right)_{0}(w),
$$

which after integration by parts is equivalent to

$$
\sum_{I=1}^{h} \oint_{A_{I}}\left(\hat{\omega}_{+}\right)_{0} \Sigma_{I}(z, w)=2 \pi\left(\hat{\omega}_{+}\right)_{0}(w) .
$$

We observe that both conditions (6.21) and (6.22) are manifestly satisfied by (6.16) to first order in $\chi_{\bar{z}}^{+}$.

Returning to super-abelian differentials, we could look for solutions of the equation $\mathscr{D}_{-}^{1 / 2} \hat{\omega}=0$ which translates in component language into the system (5.7). At first sight it would seem that this system should admit $h+1$ solutions, to be generated perturbatively from the solutions at $\chi=0$ given by $\theta \omega_{I}, I=1, \ldots, h$ and $h_{\delta}$. However for $\chi$ different from 0 , solvability of the first equation in (5.8) imposes a constraint

$$
\int d^{2} z h_{\delta} \chi_{\bar{z}}^{+} \hat{\omega}_{z}=0
$$

which is not satisfied generically. From this point of view, the super Riemann surfaces with $\chi_{\bar{z}}^{+}=0$ constitute a singular variety about which we cannot expand perturbatively, and generically there will be only one single-valued superholomorphic super abelian differential, the one which generalizes the Dirac zero mode.

Conformal field theory suggests on the other hand to allow for superholomorphic differentials with non-trivial monodromy. The monodromy should only cancel out in the end for the full amplitude. That super-holomorphic differentials of this more general type do exist is evident from the fact that allowing for non-trivial monodromy, the Dirac operator with odd spin structure is still invertible, and its inverse is given by the meromorphic propagator $S_{\delta}(z, w)$ of $(6.5)$. Although there is great freedom in defining these notions, the choices we shall make are dictated by the structure of the chiral amplitudes. The situation becomes then similar to the case of even spin structure, and there are indeed $h+1$ super- 
abelian differentials, $h$ of which are odd super-fields (analogues of the ordinary abelian differentials), and one of which is even (analogue of the Dirac zero mode). More explicitly, they are defined by

$$
\begin{aligned}
& \hat{\omega}_{I}=\left(\hat{\omega}_{+}\right)_{I}+\theta\left(\hat{\omega}_{z}\right)_{I}, \\
& \hat{\omega}_{0}=\left(\hat{\omega}_{+}\right)_{0}+\theta\left(\hat{\omega}_{z}\right)_{0}
\end{aligned}
$$

with the following perturbative expansions for the components of the odd supermultiplet

$$
\begin{aligned}
\left(\hat{\omega}_{z}\right)_{I}(z) & =\omega_{I}(z)+\frac{1}{4 \pi} \int d^{2} y \chi_{\bar{y}}^{+} \partial_{z} \partial_{y} \ln E(y, z)\left(\hat{\omega}_{+}\right)_{I}(y), \\
\left(\hat{\omega}_{+}\right)_{I}(z) & =-\frac{1}{4 \pi} \int d^{2} y \chi_{\bar{y}}^{+} \hat{S}_{\delta}(z, y) \omega_{I}(y),
\end{aligned}
$$

and for the even supermultiplet

$$
\begin{aligned}
\left(\hat{\omega}_{+}\right)_{0}(z) & =h_{\delta}(z)+\frac{1}{4 \pi} \int d^{2} y \hat{S}_{\delta}(z, y) \chi_{\bar{y}}^{+}\left(\hat{\omega}_{z}\right)_{0}(y), \\
\left(\hat{\omega}_{z}\right)_{0}(z) & =\frac{1}{4 \pi} \int d^{2} w \partial_{z} \partial_{w} \ln E(z, w) \chi_{\bar{w}}^{+}\left(\hat{\omega}_{+}\right)_{0}(w)
\end{aligned}
$$

It should be noted that all these forms depend on the choice of curves along which the surface is cut, and not just on the choice of basis for the homology group. There is also an arbitrary normalization in $\hat{\omega}_{0}$ not related to the homology. We have chosen it so that $\hat{\omega}_{0}$ reduces to $h_{\delta}$ when $\chi_{\bar{z}}^{+}=0$.

Next we list out all the monodromies of the super abelian differentials. It is easily seen that the $\left(\hat{\omega}_{+}\right)_{0}$ introduced above coincides precisely with the object introduced in (6.17). We have already shown there that $\left(\hat{\omega}_{+}\right)_{0}$ is a half-form without monodromy. From (6.25) it follows that the entire half-form $\hat{\omega}_{0}$ has no monodromy either, in agreement with the general discussion in terms of the constraint (6.23). From the monodromy of $\hat{S}_{\delta}$ in $(6.14)$, we can read off the monodromies of the other super abelian differentials

$$
\begin{array}{ll}
\left(\hat{\omega}_{z}\right)_{I}\left(z+A_{K}\right)=\left(\hat{\omega}_{z}\right)_{I}(z), & \left(\hat{\omega}_{z}\right)_{I}\left(z+B_{K}\right)=\left(\hat{\omega}_{z}\right)_{I}(z), \\
\left(\hat{\omega}_{+}\right)_{I}\left(z+A_{K}\right)=\left(\hat{\omega}_{+}\right)_{I}(z), & \left(\hat{\omega}_{+}\right)_{I}\left(z+B_{K}\right)=\left(\hat{\omega}_{+}\right)_{I}(z)+\frac{i}{2 \pi} h_{\delta} \hat{V}_{K} \hat{\Omega}_{I 0},
\end{array}
$$

where

$$
\hat{\Omega}_{I 0}=\frac{i}{2} \int \omega_{I}(w) \chi_{\bar{w}}^{+}\left(\hat{\omega}_{+}\right)_{0}(w) .
$$

To evaluate the periods, we can just use the formula (5.21) which is valid even in presence of monodromy for the forms $\phi_{\bar{z}}$. The result is

$$
\begin{aligned}
\oint_{A_{I}} \hat{\omega}_{J} & =\delta_{I J}+\frac{i}{8 \pi} \int d^{2} w \int_{P}^{P+A_{I}} \partial_{z} \partial_{w} \ln E(z, w)\left(\chi_{\bar{w}}^{+}\left(\hat{\omega}_{+}\right)_{I}(w)\right) \\
& =\delta_{I J}, \\
\oint_{B_{I}} \hat{\omega}_{J} & =\Omega_{I J}+\frac{1}{4 \pi} \int d^{2} w \int_{P}^{P+B_{I}} \partial_{z} \partial_{w} \ln E(z, w)\left(\chi_{\bar{w}}^{+}\left(\hat{\omega}_{+}\right)_{J}(w)\right) \\
& =\Omega_{I J}+\frac{i}{2} \int d^{2} w \omega_{I}(w) \chi_{\bar{w}}^{+}\left(\hat{\omega}_{+}\right)_{J} .
\end{aligned}
$$


This means that the super period matrix can be taken as the expression on the last line of the above equations, and put in symmetric form

$$
\hat{\Omega}_{I J}=\Omega_{I J}-\frac{1}{4 \pi} \int d^{2} z d^{2} w \chi_{\bar{z}}^{+} \omega_{I}(z) \hat{S}_{\delta}(z, w) \chi_{\bar{w}}^{+} \omega_{J}(w)
$$

by the same iteration arguments as in (5.24)-(5.26) for the even spin structure case. Analogously one finds

$$
\oint_{A_{I}} \hat{\omega}_{0}=0, \quad \oint_{B_{I}} \hat{\omega}_{0}=\hat{\Omega}_{I 0}
$$

with $\hat{\Omega}_{I 0}$ the vector given by (6.27). It should be noted that since these super abelian differentials were defined relative to a cut surface, the super period matrix depends as well on these choices. This dependence will cancel out in physical amplitudes.

Finally it is to be expected that we shall also require a generalization of the super prime form to odd spin structure, in analogy to (5.18):

$$
\begin{aligned}
-\ln \mathscr{E}_{\delta}(\mathbf{z}, \mathbf{w})= & -\ln E(z, w)+\theta_{z} \theta_{w} \hat{S}_{\delta}(z, w) \\
& +\frac{1}{4 \pi} \theta_{z} \int d^{2} y \chi_{\bar{y}}^{+} \partial_{y} \ln E(y, w) \hat{S}_{\delta}(z, y) \\
& +\frac{1}{4 \pi} \theta_{w} \int d^{2} y \chi_{\bar{y}}^{+} \partial_{y} \ln E(y, z) \hat{S}_{\delta}(w, y) \\
& +\frac{1}{16 \pi^{2}} \int d^{2} x d^{2} y \chi_{\bar{x}}^{+} \partial_{x} \ln E(x, z) \chi_{\bar{y}}^{+} \partial_{y} \ln E(y, w) \hat{S}_{\delta}(x, y) .
\end{aligned}
$$

The above integrals should be viewed as carried out over the cut Riemann surface. As in the case of even spin structure, $\ln \mathscr{E}_{\delta}$ is defined through chiral amplitudes only up to an additive function $f(\mathbf{z})+f(\mathbf{w})$. Moreover we shall establish later on that the presence of the Dirac zero mode also allows a redefinition of the form

$$
\ln \mathscr{E}_{\delta}(z, \mathbf{w}) \rightarrow \ln \mathscr{E}_{\delta}(\mathbf{z}, \mathbf{w})+f(\mathbf{z})+f(\mathbf{w})+g(\mathbf{z}) \int^{\mathbf{w}} \hat{\omega}_{0}+g(\mathbf{w}) \int^{\mathbf{z}} \hat{\omega}_{0}
$$

where $f(\mathbf{z})$ and $g(\mathbf{z})$ are arbitrary functions dependent only on $\mathbf{z}$ and possibly exhibiting non-trivial monodromy. It is easy to see that

$$
\mathscr{D}_{-}^{0} \partial_{+}^{w} \ln \mathscr{E}_{\delta}(\mathbf{z}, \mathbf{w})=2 \pi \bar{\theta}_{z}\left(\theta_{z}-\theta_{w}\right) \delta(z-w),
$$

where the right-hand side can be viewed as the Dirac delta function on super-fields dependent only on $\theta$ and not on $\bar{\theta}$. If this property is to be preserved under redefinitions by $f$ and $g$ functions as above, we have to restrict to

$$
\mathscr{D}-g=0 \text {. }
$$


By analogy with the case of even spin structures, we shall add functions $f$ as in (5.37), so as to obtain monodromies close to those of that case and the bosonic case:

$$
\begin{aligned}
-\ln \mathscr{E}_{\delta}(\mathbf{z}, \mathbf{w})= & -\ln E(z, w)+\theta_{z} \theta_{w} \hat{S}_{\delta}(z, w) \\
& +\frac{1}{4 \pi} \theta_{z} \int d^{2} y \chi_{\bar{y}}^{+} \partial_{y} \ln \frac{E(y, w)}{E(y, z)} \hat{S}_{\delta}(z, y) \\
& +\frac{1}{4 \pi} \theta_{w} \int d^{2} y \chi_{\bar{y}}^{+} \partial_{y} \ln \frac{E(y, z)}{E(y, w)} \hat{S}_{\delta}(w, y) \\
& +\frac{1}{32 \pi^{2}} \int d^{2} x d^{2} y \chi_{\bar{x}}^{+} \partial_{x} \ln \frac{E(x, z)}{E(x, w)} \chi_{\bar{y}}^{+} \partial_{y} \ln \frac{E(y, w)}{E(y, z)} \hat{S}_{\delta}(x, y) \\
& +f(\mathbf{z})+f(\mathbf{w})+g(\mathbf{z}) \iint^{\mathbf{w}} \hat{\omega}_{0}+g(\mathbf{w}) \int^{\mathbf{z}} \hat{\omega}_{0} .
\end{aligned}
$$

This expression is still not properly defined, since $\hat{S}_{\delta}$ has non-trivial monodromy. Taking $\hat{S}_{\delta}$ as in (6.14) defines a particular super prime form with monodromy given by

$$
\begin{aligned}
\mathscr{E}_{\delta}\left(\mathbf{z}+A_{K}, \mathbf{w}\right) & =\mathscr{E}_{\delta}(\mathbf{z}, \mathbf{w}), \\
\mathscr{E}_{\delta}\left(\mathbf{z}+B_{K}, \mathbf{w}\right) & =\mathscr{E}_{\delta}(\mathbf{z}, \mathbf{w}) \exp \left(-i \pi \hat{\Omega}_{K K}-2 \pi i \int_{\mathbf{w}}^{\mathbf{z}} \hat{\omega}_{K}+\theta_{z} h_{\delta}(z) \hat{V}_{K} \int_{\mathbf{w}}^{\mathbf{z}} \hat{\omega}_{0}\right), \\
\partial_{+}^{w} \ln \mathscr{E}_{\delta}\left(\mathbf{z}+A_{K}, \mathbf{w}\right) & =\partial_{+}^{\mathbf{w}} \ln \mathscr{E}_{\delta}(\mathbf{z}, \mathbf{w}), \\
\partial_{+}^{w} \ln \mathscr{E}_{\delta}\left(\mathbf{z}+B_{K}, \mathbf{w}\right) & =\partial_{+}^{w} \ln \mathscr{E}_{\delta}(\mathbf{z}, \mathbf{w})+2 \pi i \hat{\omega}_{K}(\mathbf{w})+\theta_{z} h_{\delta}(z) \hat{V}_{K} \hat{\omega}_{0}(\mathbf{w}), \\
\partial_{+}^{\mathbf{z}} \partial_{+}^{\mathrm{w}} \ln \mathscr{E}_{\delta}\left(\mathbf{z}+A_{K}, \mathbf{w}\right) & =\partial_{+}^{\mathbf{z}} \partial_{+}^{\mathrm{w}} \ln \mathscr{E}_{\delta}(\mathbf{z}, \mathbf{w}), \\
\partial_{+}^{\mathbf{z}} \partial_{+}^{\mathrm{w}} \ln \mathscr{E}_{\delta}\left(\mathbf{z}+B_{K}, \mathbf{w}\right) & =\partial_{+}^{\mathbf{z}} \partial_{+}^{\mathrm{w}} \ln \mathscr{E}_{\delta}(\mathbf{z}, \mathbf{w})+h_{\delta}(z) \hat{V}_{K} \hat{\omega}_{0}(\mathbf{w}) .
\end{aligned}
$$

The super prime form for odd spin structure shares a number of further properties with the ordinary prime form:

$$
\left\{\begin{array}{r}
\mathscr{E}_{\delta}(\mathbf{z}, \mathbf{w})=0 \\
\mathscr{D}_{+}^{\mathbf{z}} \mathscr{E}_{\delta}(\mathbf{z}, \mathbf{w})=0
\end{array}\right\} \Leftrightarrow\left\{\begin{array}{c}
z=w \\
\theta_{z}=\theta_{w}
\end{array}\right\} .
$$

(ii) $\mathscr{E}_{\delta}(\mathbf{z}, \mathbf{w})$ is a $(-1 / 2,0) \times(-1 / 2,0)$ form in $\mathbf{z}$ and $\mathbf{w}$.

(iii) $\mathscr{E}_{\delta}(\mathbf{z}, \mathbf{w})$ is holomorphic in all its arguments.

We may now rewrite the full chiral amplitudes for scalar superfields in terms of the above superconformal invariants of the super Riemann surface. First the source independent part of $C_{\delta}^{m}$ is obtained from (6.13) by setting $k=\zeta=p=\psi_{+}^{0, \mu}=0$,

$$
Z_{\mathscr{D}, \delta}^{-1}=Z_{\Delta}(\Omega)^{-1} Z_{\delta}(\Omega)\left\langle\exp \left[-\frac{1}{4 \pi} \int d^{2} z \chi_{\bar{z}}^{+} \psi_{+} \partial_{z} x_{+}\right]\right\rangle_{x_{+}, \psi_{+}} .
$$

The $\psi_{+}$contractions are performed with the propagator $S_{\delta}(z, w)$. Since any function of $\Omega$ and $\chi_{\bar{z}}^{+}$can be expressed as a function of $\hat{\Omega}$ and $\chi_{\bar{z}}^{+}$and vice versa, the 
quantity $Z_{\mathscr{D}, \delta}$ should be thought of as a function of $\hat{\Omega}$ and $\chi_{\bar{z}}^{+}$. Returning to (6.13) we can carry out the $x_{+}$contractions, obtaining

$$
\begin{aligned}
& C_{\delta}^{m}= \\
& \left\langle\exp \left[i \pi p_{I}^{\mu} \Omega_{I J} p_{J}^{\mu}+2 \pi p_{I}^{\mu}\left(\zeta_{i} \theta_{i} \omega_{I}\left(z_{i}\right)+i k_{i}^{\mu} \int_{P}^{z_{i}} \omega_{I}-\frac{1}{4 \pi} \int d^{2} z \chi_{\bar{z}}^{+} \psi_{+} \omega_{I}(z)\right)\right]\right. \\
& \quad \times \prod_{i=1}^{n} \exp \left[\left(i k_{i} \theta_{i}+\zeta_{i}\right) \psi_{+}\left(z_{i}\right)-\frac{1}{4 \pi}\left(\zeta_{i} \theta_{i} \partial_{z_{i}}+i k_{i}\right) \int d^{2} z \chi_{\bar{z}}^{+} \psi_{+} \partial_{z} \ln E\left(z, z_{i}\right)\right] \\
& \quad \times \exp \left[\frac{1}{16 \pi^{2}}\left(\int d^{2} z d^{2} w \chi_{\bar{z}}^{+} \psi_{+} \partial_{z} \partial_{w} \ln E(z, w) \chi_{\bar{w}}^{+} h_{\delta}(w)\right) \psi_{+}^{0}\right] \\
& \quad \times \exp \left[\left(\left(i k_{i} \theta_{i}+\zeta_{i}\right) h_{\delta}\left(z_{i}\right)-\frac{1}{4 \pi}\left(\zeta_{i} \theta_{i} \partial_{z_{i}}+i k_{i}\right) \int d^{2} z \chi_{\bar{z}}^{+} h_{\delta} \partial_{z} \ln E\left(z, z_{i}\right)\right.\right. \\
& \left.\left.\left.\quad-\frac{1}{2} p_{I}^{\mu} \int d^{2} z \chi_{\bar{z}}^{+} h_{\delta} \omega_{I}(z)\right) \psi_{+}^{0}\right]\right\rangle_{\psi+} \\
& \quad \times\left\langle\prod_{i=1}^{n} \exp \left(\zeta_{i} \theta_{i} \partial_{z_{i}}+i k_{i}\right) x_{+}\left(z_{i}\right)\right\rangle_{x_{+}} .
\end{aligned}
$$

Next we contract the term

$$
-p_{I}^{\mu} \int d^{2} z \chi_{\bar{z}}^{+} \psi_{+} \omega_{I}(z) / 2 .
$$

This shifts all the abelian differentials and period matrix in the above expression to their super analogues as defined in (6.25)-(6.30),

$$
\begin{aligned}
C_{\delta}^{m}= & \left\langle\exp \left[i \pi p_{I}^{\mu} \hat{\Omega}_{I J} p_{J}^{\mu}+2 \pi p_{I}^{\mu}\left(i k_{i}+\zeta_{i} \partial_{+}\right) \int^{z_{i}, \theta_{i}} d \mathbf{z}_{i} \hat{\omega}_{I}\right]\right. \\
& \times \prod_{i=1}^{n} \exp \left[\left(i k_{i} \theta_{i}+\zeta_{i}\right) \psi_{+}\left(z_{i}\right)-\frac{1}{4 \pi}\left(\zeta_{i} \theta_{i} \partial_{z_{i}}+i k_{i}\right) \int d^{2} z \chi_{\bar{z}}^{+} \psi_{+} \partial_{z} \ln E\left(z, z_{i}\right)\right. \\
& \left.+\frac{1}{16 \pi^{2}} \int d^{2} z d^{2} w \chi_{\bar{z}}^{+} \psi_{+} \partial_{z} \partial_{w} \ln E(z, w) \chi_{\bar{w}}^{+} h_{\delta}(w) \psi_{+}^{0}\right] \\
& \times\left\langle\prod_{i=1}^{n} \exp \left[\left(\zeta_{i} \theta_{i} \partial_{z_{i}}+i k_{i}\right) x_{+}\left(z_{i}\right)\right]\right\rangle_{x_{+}} \\
& \times \exp \left[\left(\left(i k_{i} \theta_{i}+\zeta_{i}\right) h_{\delta}\left(z_{i}\right)-\frac{1}{4 \pi}\left(\zeta_{i} \theta_{i} \partial_{z_{i}}+i k_{i}\right) \int d^{2} z \chi_{\bar{z}}^{+} h_{\delta} \partial_{z} \ln E\left(z, z_{i}\right)\right.\right. \\
& \left.\left.\left.-\frac{1}{2} p_{I}^{\mu} \int d^{2} z \chi_{\bar{z}}^{+}\left(\hat{\omega}_{+}\right)_{0} \omega_{I}(z)\right) \psi_{+}^{0}\right]\right\rangle_{\psi+} .
\end{aligned}
$$

A final partial contraction with the term involving both $\psi_{+}$and $\psi_{+}^{0}$ above will shift the $h_{\delta}$ terms in the remaining $\psi_{+}^{0}$ factors to $\left(\hat{\omega}_{+}\right)_{0}$. The net answer takes the 
following simple form in terms of super abelian differentials and super period matrix

$$
\begin{aligned}
& C_{\delta}^{m}\left(\mathbf{z}_{i}, \zeta_{i}^{\mu}, k_{i}^{\mu} ; \hat{\Omega}, \chi_{\bar{z}}^{+} ; \psi_{+}^{0, \mu}, p_{I}^{\mu}\right)=Z_{\mathscr{D}, \delta}^{-10}\left(\hat{\Omega}, \chi_{\bar{z}}^{+}\right) \exp \left[i \pi p_{I}^{\mu} \hat{\Omega}_{I J} p_{J}^{\mu}+2 \pi i p_{I}^{\mu} \hat{\Omega}_{I 0} \psi_{+}^{0, \mu}\right] \\
& \quad \times \exp \left[2 \pi \sum_{i=1}^{n}\left(i k_{i}^{\mu}+\zeta_{i}^{\mu} \partial_{+}^{i}\right) \int_{P}^{z_{i}, \theta_{l}}\left(p_{I}^{\mu} \hat{\omega}_{I}+\psi_{+}^{0, \mu} \hat{\omega}_{0}\right)\right] \\
& \quad \times\left\langle\exp \left[i k_{i}^{\mu} X_{+}^{\mu}\left(\mathbf{z}_{i}\right)+\zeta_{i}^{\mu} \partial_{+} X_{+}^{\mu}\left(\mathbf{z}_{i}\right)\right]\right\rangle_{X_{+}} .
\end{aligned}
$$

The last expectation value can be evaluated in terms of the super prime form, exactly as in (5.44),

$$
\begin{aligned}
& \left\langle\exp \left(i k_{i} X_{+}^{\mu}\left(\mathbf{z}_{i}\right)+\zeta_{i}^{\mu} \partial_{+}^{i} X_{+}^{\mu}\left(\mathbf{z}_{i}\right)\right)\right\rangle \\
& \quad=\exp \left(\sum_{i, j}\left(\frac{1}{2} k_{i} k_{j}+\frac{1}{2} \zeta_{i} \zeta_{j} \partial_{+}^{i} \partial_{+}^{j}-i k_{i} \zeta_{j} \partial_{+}^{j}\right) \ln \mathscr{E}_{\delta}\left(\mathbf{z}_{i}, \mathbf{z}_{j}\right)\right) .
\end{aligned}
$$

Under redefinitions of the super prime form by (6.32) this picks up a factor

$$
\exp \left(\sum_{i, j}\left(-\frac{i}{2} k_{i}^{\mu} g\left(\mathbf{z}_{i}\right)-\frac{1}{2} \zeta_{i} \partial_{+}^{i} g\left(\mathbf{z}_{i}\right)\right)\left(i k_{j}^{\mu} \int^{\mathbf{z}_{j}} \hat{\omega}_{0}+\zeta_{j} \partial_{+}^{j} \int^{\mathbf{z}_{j}} \hat{\omega}_{0}\right)\right),
$$

which in (6.13) amounts to shifting $\psi_{+}^{\mu, 0}$ by

$$
\psi_{+}^{\mu, 0} \rightarrow \psi_{+}^{\mu, 0}+\frac{1}{4 \pi} \sum_{j}\left(i g\left(\mathbf{z}_{j}\right) k_{j}^{\mu}+\zeta_{j}^{\mu} \partial_{+}^{j} g\left(\mathbf{z}_{j}\right)\right) .
$$

This clearly leaves the full amplitude $\mathscr{F}_{\delta}^{m}$ unchanged.

It is useful to determine the precise relation between the super determinant of the super Laplacian on scalars and the determinants of the usual Laplacian and Dirac operator in component language. For odd spin structure this is achieved by integrating out the internal momenta $p_{I}^{\mu}$ and the Dirac zero mode $\psi_{+}^{0, \mu}$. In this way we obtain

$$
\left(\frac{s \operatorname{det}^{\prime} \mathscr{D}_{+} \mathscr{D}_{-}^{(0)}}{\int d^{2} \mathbf{z} E}\right)^{-1}=\left|Z_{\mathscr{D}, \delta}\right|^{-2} e^{-2 S_{s L}}\left(\operatorname{det} \operatorname{Im} \hat{\Omega}_{I J}\right)^{-1 / 2}\left(\hat{\Omega}_{I 0}\left(\operatorname{Im} \hat{\Omega}_{I J}^{-1} \hat{\Omega}_{J 0}\right),\right.
$$

where the prime stands for the omission of the constant zero mode only. In superstring theory we integrate also over the supergeometries, parametrized by supermoduli (see Sect. 7 below). The above expression will thus contribute to the superstring amplitude only if the Dirac zero modes are absorbed by the supermoduli. This can only be the case when the dimension of space-time (which is the number of scalar superfields) is larger than the genus of the surface. So in $\mathrm{d}=10$, the odd spin structure starts contributing to the superstring partition function only at genus $\mathrm{h}=10$.

We conclude this section with a discussion of geometric invariants in the case of odd spin structures. As shown in the beginning of the section, the amplitudes $\mathscr{F}_{\delta}^{m}$ are chiral with manifest holomorphic dependence on the supermoduli parameters, and meromorphic dependence on the vertex operator insertion points. We have also succeeded in expressing it in terms of quantities such as super abelian differentials, super period matrix and super prime form, which are 
formally supergeometric. Even though individually these notions depend on a choice of cuts, the amplitude $\mathscr{F}_{\delta}^{m}$ is independent of these choices. We shall now argue that completely intrinsic versions of these objects exist, in terms of which $\mathscr{F}_{\delta}^{m}$ can be expressed in a similar way.

We begin with an intrinsic version of $\partial_{+}^{w} \ln \mathscr{E}_{\delta}$ whose monodromy should be of the form

$$
\begin{aligned}
& \partial_{+}^{\mathrm{w}} \ln \mathscr{E}_{\delta}\left(\mathbf{z}+A_{K}, \mathbf{w}\right)=\partial_{+}^{\mathrm{w}} \ln \mathscr{E}_{\delta}(\mathbf{z}, \mathbf{w}), \\
& \partial_{+}^{\mathrm{w}} \ln \mathscr{E}_{\delta}\left(\mathbf{z}+B_{K}, \mathbf{w}\right)=\partial_{+}^{\mathrm{w}} \ln \mathscr{E}_{\delta}(\mathbf{z}, \mathbf{w})+2 \pi i \hat{\omega}_{K}(\mathbf{w})+\int^{\mathbf{z}} \hat{\omega}_{0} \hat{W}_{K} \hat{\omega}_{0}(\mathbf{w}),
\end{aligned}
$$

with

$$
\hat{W}_{K}=\frac{W_{K}}{\sum_{I=1}^{h} W_{I} \oint_{A_{I}} \hat{\omega}_{0}(\mathbf{w}) \int^{\mathbf{w}} \hat{\omega}_{0}}
$$

for an arbitrary vector $W_{I}$ in the Jacobian. These monodromies are characterized by the fact that they are superholomorphic, in agreement with the equation satisfied by $\partial_{+}^{\mathrm{w}} \ln \mathscr{E}_{\delta}$,

$$
\mathscr{D}_{-}^{0} \partial_{+}^{w} \ln \mathscr{E}_{\delta}(\mathbf{z}, \mathbf{w})=2 \pi \bar{\theta}_{z}\left(\theta_{z}-\theta_{w}\right) \delta(z-w),
$$

and have themselves monodromies no worse than those of $\hat{\omega}_{K}$. Furthermore they satisfy the key consistency requirement on the monodromy of $\partial_{+}^{w} \ln \mathscr{E}_{\delta}$ which follows from orthogonality to the only half-form without monodromy, namely $\hat{\omega}_{0}$. In fact integrating (6.45) versus $\hat{\omega}_{0}$ gives

$$
\int \mathscr{D}_{-}\left(\hat{\omega}_{0}(\mathbf{z}) \partial_{+}^{w} \ln \mathscr{E}_{\delta}(\mathbf{z}, \mathbf{w})\right)=2 \pi \hat{\omega}_{0}(\mathbf{w}) .
$$

The left-hand side of (6.46) can be evaluated in terms of the monodromy alone, and we get

$$
\sum_{K=1}^{h} \oint_{A_{K}} \hat{\omega}_{0}(\mathbf{z}) \partial_{+}^{w} \ln \frac{\mathscr{E}_{\delta}\left(\mathbf{z}+B_{K}, \mathbf{w}\right)}{\mathscr{E}_{\delta}(\mathbf{z}, \mathbf{w})}=2 \pi
$$

The monodromy prescribed in (6.43)-(6.44) does satisfy this equation. The general theory of equations tells us that if the consistency requirements are satisfied the solution should exist. From the monodromy of $\partial_{+}^{w} \ln \mathscr{E}_{\delta}$ we can deduce the monodromy of $\mathscr{E}_{\delta}$ itself, as well as those of the super abelian differentials

$$
\begin{aligned}
& \mathscr{E}_{\delta}\left(\mathbf{z}+A_{K}, \mathbf{w}\right)=\mathscr{E}_{\delta}(\mathbf{z}, \mathbf{w}), \\
& \mathscr{E}_{\delta}\left(\mathbf{z}+B_{K}, \mathbf{w}\right)=\mathscr{E}_{\delta}(\mathbf{z}, \mathbf{w}) \exp \left(-i \pi \hat{\Omega}_{K K}+2 \pi i \int_{\mathbf{z}}^{\mathrm{w}} \hat{\omega}_{K}+\int^{\mathrm{z}} \hat{\omega}_{0} \hat{W}_{K} \int^{\mathrm{w}} \hat{\omega}_{0}\right) .
\end{aligned}
$$

Similarly the super abelian differentials can be written as

$$
2 \pi i \hat{\omega}_{K}(\mathbf{w})=\oint_{B_{K}} \partial_{+}^{\mathbf{z}} \partial_{+}^{\mathbf{w}} \ln \mathscr{E}_{\delta}(\mathbf{z}, \mathbf{w}),
$$

and thus their monodromy is given by

$$
\begin{aligned}
& \hat{\omega}_{K}\left(\mathbf{w}+A_{L}\right)=\hat{\omega}_{K}(\mathbf{w}), \\
& \hat{\omega}_{K}\left(\mathbf{w}+B_{L}\right)=\hat{\omega}_{K}(\mathbf{w})+\hat{\Omega}_{K 0} \hat{W}_{L} \hat{\omega}_{0}(\mathbf{w}) .
\end{aligned}
$$


We note that the monodromies derived in this manner are consistent with the constraint

$$
0=\int \mathscr{D}_{-} \hat{\omega}_{L}=\sum_{K=1}^{h} \oint_{A_{K}} \hat{\omega}_{0} \hat{\Omega}_{L 0} \hat{W}_{K} .
$$

It would of course be very valuable to have explicit expressions for all these quantities. We expect then the same formula (6.40) for $\mathscr{F}_{\delta}^{m}$ to hold with these intrinsic supergeometric invariants.

\section{Chiral Superstring Scattering Amplitudes}

With the above completely explicit description of correlation functions of scalar superfields on super Riemann surfaces, it is now easy to clarify the chiral structure of superstring scattering amplitudes.

The action for superstring propagation is obtained by coupling 10 scalar superfields $X^{\mu}$ to two-dimensional supergravity $E_{M}{ }^{A}$. In the Polyakov formulation of Type II superstring theory, the amplitude for the scattering of on-shell physical particles will be constructed out of the correlation function

$$
\langle\Theta\rangle_{I I}=\sum_{h=0}^{\infty} \int D E_{M}{ }^{A} D X^{\mu} \Theta e^{-I_{m}},
$$

where one sums over all topologies $(h)$ of underlying Riemann surfaces and all supergeometries satisfying the torsion constraints (3.3), as well as over all embeddings $X^{\mu}$. The Gliozzi-Scherk-Olive projection in the functional formalism requires that for each Riemann surface, the scalar superfield integrals be carried out with respect to a fixed spin structure, upon which contributions of left and right movers are separated, given independent spin structures which are to be summed over. Our goal is to give a precise and workable version of this prescription. The operator $\Theta$ is the product of $n$ vertex operators of the form (3.6), or in the case of massless bosons, of the form (3.7), constructed in such a way as to be $s \operatorname{Diff}(M)$ and $s$ Weyl $(M)$ invariant. Once these invariances are guaranteed, the integration over all supergeometries reduces to an integral over the equivalence classes under these symmetries of the supergeometries, or supermoduli space $s \mathscr{M}_{h}$ :

$$
s \mathscr{M}_{h}=\frac{\left\{E_{M}{ }^{A}, \Omega_{M} \text { satisfying }(3.3)\right\}}{s \operatorname{Diff}(M) \times s \operatorname{Weyl}(M)}
$$

with graded complex dimensions

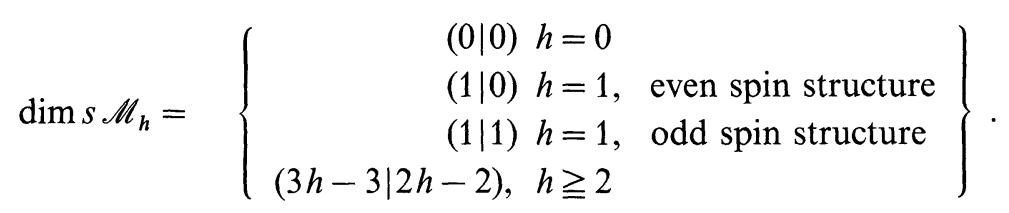

In [10] it was shown that supermoduli space is a complex super-manifold, whose complex structure is naturally induced by the one on the supergeometry. After gauge-fixing a slice $\hat{E}_{M}{ }^{A}\left(m_{J}\right)$ for $s \mathscr{M}_{h}$, the integration over supergeometries can be 
recast in terms of an integral over supermoduli parameters $m_{\boldsymbol{J}}$ and superghost fields $B$ and $C$ of $U(1)$ weights $3 / 2$ and -1 respectively:

$$
\langle\Theta\rangle_{I I}=\sum_{h=0}^{\infty} \int_{s \mathscr{M}_{h}} \prod_{J=1}^{5 h-5} d^{2} m_{J} D\left(B \bar{B} C \bar{C} X^{\mu}\right)\left|\delta\left(\left\langle\mu_{J} \mid B\right\rangle\right)\right|^{2} \Theta e^{-I_{m}-I_{s g h}}
$$

with the superghost action given by

$$
I_{s g h}(B, C)=\frac{1}{2 \pi} \int d^{2} \mathbf{z} E\left(B \mathscr{D}-C+\bar{B} \mathscr{D}_{+} \bar{C}\right)
$$

and $\mu_{J}$ is the super-Beltrami differential of the slice $\hat{E}_{M}{ }^{A}$

$$
\left(\mu_{J}\right)_{-}{ }^{z}=(-)^{(m+1) j} \hat{E}_{-}{ }^{M} \frac{\partial \hat{E}_{M}{ }^{z}}{\partial m_{J}} .
$$

It is clear that the superghost action is split into a holomorphic function $\mathscr{F}_{\delta}^{\text {sgh }}$ on supermoduli, times its complex conjugate, up to a local anomaly which cancels that of the matter fields in the critical dimension $d=10$. Thus up to the local anomaly

$$
\int D(B C) \prod_{J=1}^{5 h-5} \delta\left(\left\langle\mu_{J} \mid B\right\rangle\right) e^{-\frac{1}{2 \pi} \int d^{2} \mathbf{z} E B \mathscr{D}_{-} C}=\mathscr{F}_{\delta}^{s g h}\left(\hat{\Omega}, \chi_{\bar{z}}^{+}\right)
$$

\section{Massless Bosonic Particles}

We can combine this contribution of the superghosts with the chirally split contributions of the scalar superfields in (5.4) and (6.13) to produce the manifestly split, anomaly-free superstring amplitudes for massless external bosonic particles,

$$
\begin{aligned}
& \left\langle\prod_{i=1}^{n} \exp \left[i k_{i}^{\mu} X^{\mu}\left(\mathbf{z}_{i}\right)+\zeta_{i}^{\mu} \sqrt{E} \mathscr{D}_{+} X^{\mu}\left(\mathbf{z}_{i}\right)+\bar{\zeta}_{i}^{\mu} \sqrt{E} \mathscr{D}_{-} X^{\mu}\left(\mathbf{z}_{i}\right)\right]\right\rangle \\
& \quad=\sum_{h=0}^{\infty} \int d p_{I}^{\mu} \int_{s \mathscr{M}_{h}} \prod_{J=1}^{5 h-5} d^{2} m_{J} f_{S}\left(\mathbf{z}_{i}, \zeta_{i}^{\mu}, k_{i}^{\mu} ; \hat{\Omega}, \chi_{\bar{z}}^{+} ; p_{I}^{\mu}\right) \overline{f_{S}\left(\mathbf{z}_{i}, \zeta_{i}^{\mu}, k_{i}^{\mu} ; \hat{\Omega}, \chi_{\bar{z}}^{+} ; p_{I}^{\mu}\right)} .
\end{aligned}
$$

Here

$$
\begin{aligned}
f_{S}\left(\mathbf{z}_{i} ; \zeta_{i}^{\mu}, k_{i}^{\mu} ; \hat{\Omega}, \chi_{\bar{z}}^{+} ; p_{I}^{\mu}\right)= & \sum_{\delta \text { even }} \mathscr{F}_{\delta}^{s g h}\left(\hat{\Omega}, \chi_{\bar{z}}^{+}\right) \mathscr{F}_{\delta}^{m}\left(\mathbf{z}_{i}, \zeta_{i}^{\mu}, k_{i}^{\mu} ; \hat{\Omega}, \chi_{\bar{z}}^{+} ; p_{I}^{\mu}\right) \\
& \mp \sum_{\delta \text { odd }} \mathscr{F}_{\delta}^{s g h}\left(\hat{\Omega}, \chi_{\bar{z}}^{+}\right) \mathscr{F}_{\delta}^{m}\left(\mathbf{z}_{i}, \zeta_{i}^{\mu}, k_{i}^{\mu} ; \hat{\Omega}, \chi_{\bar{z}}^{+} ; p_{I}^{\mu}\right) .
\end{aligned}
$$

Recall that the amplitude $\mathscr{F}_{\delta}^{m}$ involves an integral over the Dirac zero mode, as indicated in (6.13). The first term on the right-hand side of (7.7) is always parity conserving, and the second one is always parity violating since it involves a 10-dimensional $\varepsilon$ symbol due to the Dirac zero mode integration. If the relative sign between the even and the odd contributions are the same for both $f_{S}$ and $\bar{f}_{S}$, we have the Type IIA string, if they are opposite, we have the Type IIB string. 
Heterotic string amplitudes are obtained in a similar way

$$
\begin{aligned}
& \left\langle\prod_{i=1}^{n} \exp \left[i k_{i}^{\mu} X^{\mu}\left(\mathbf{z}_{i}\right)+\zeta_{i}^{\mu} \mathscr{D}_{+} X^{\mu}\left(\mathbf{z}_{i}\right)+\bar{\zeta}_{i}^{\mu} \mathscr{D}_{-} X^{\mu}\left(\mathbf{z}_{i}\right)\right]\right\rangle_{H S} \\
& =\sum_{h=0}^{\infty} \int d p_{I}^{\mu} \int_{s \mathscr{M}_{h} \times \mathscr{M}_{k}} \prod_{J=1}^{5 h-5} d m_{J} \prod_{j=1}^{3 h-3} \overline{d m_{j}} \\
& \quad \times f_{S}\left(\mathbf{z}_{i}, \zeta_{i}^{\mu}, k_{i}^{\mu} ; \hat{\Omega}, \chi_{\bar{z}}^{+}, p_{I}^{\mu}\right) \overline{f_{B}\left(z_{i}, \zeta_{i}^{\mu}, k_{i}^{\mu}, k_{i}^{\alpha}, \Omega, p_{I}^{\mu}\right)}
\end{aligned}
$$

with $f_{B}$ the chiral bosonic amplitude, and $k_{i}^{\alpha}$ the external momenta corresponding to the external gauge degrees of freedom of the heterotic string states.

\section{Arbitrary Massive Particles ${ }^{3}$}

It was shown that scattering amplitudes can be constructed out of meromorphic chiral amplitudes for tachyonic external states in [13] and for general massless bosonic states in the present paper. After the GSO projection, the tachyon no longer belongs to the superstring spectrum, so for the superstring per se, we have established the chiral splitting of massless bosonic external states. What are the properties of amplitudes for scattering of massive bosonic or fermionic states?

Though bosonic vertex operators at any fixed mass level may always be constructed by insisting on super-Weyl invariance, there is no closed formula for the general case. As a short cut, we shall use factorization properties of superstring scattering amplitudes instead, to make statements about the properties of amplitudes including massive bosons. Except when some conservation laws or superselection rules apply, any massive bosonic particle will couple to some number of external massless bosons. Indeed, a massive particle will ultimately decay into massless particles, bosons and fermions. But through loop effects, pairs of fermions again produce massless bosons, so that there will always be a coupling of any massive boson to some massless bosons. Some processes that do go through loop effects may however not occur at tree level. As an example the decay into photons of any neutral particle cannot occur to any tree level, though it does to one loop. Of course sometimes there are superselection rules, like for the Spin (32)/Z(2) heterotic string theory. The massless gauge bosons are as always in the adjoint representation, but at the first mass level, the spinor of $\operatorname{Spin}(32) / Z(2)$ can never decay into massless particles. It is stable, and hence cannot be produced by scattering massless bosons alone. It can also not be produced by scattering massless bosons and fermions.

We now claim that scattering amplitudes involving massive external states admit a chiral splitting exactly as the massless amplitudes did, in terms of chiral amplitudes that are holomorphic in supermoduli, chiral polarization tensors, and meromorphic in vertex insertion points. To show this, let us assume at first that the massive particles we wish to scatter can be produced at tree level from massless particles. It will arise as the amplitude in which the massless particles are all close together and the massive boson vertex operator will arise in the operator product

${ }^{3}$ We thank S. Mandelstam for discussions on these issues 
expansion of the massless operators. Now the operator product expansion proceeds independently for meromorphic and anti-meromorphic fields, so that the left chiral polarization tensor for the massive state is built only out of the left chiral polarization tensors of the massless states. By construction the massive amplitude is still chirally split, i.e. can be separated into a chiral amplitude and its complex conjugate at fixed momenta, depending holomorphically on supermoduli and polarization tensors, meromorphically on insertion points. If the massive bosons to be scattered cannot be produced from massless bosons at tree level, then factorization has to proceed by letting the Riemann surface degenerate into two disconnected Riemann surfaces. The degeneration will maintain the separation between the two chiralities, so that the polarization tensor for the massive state only involves the chiral polarization tensors of the scattered massless particles. Thus the analyticity is maintained at higher mass level.

The construction of amplitudes involving space-time fermions cannot proceed along these lines. To show the chiral splitting property, one would have to start from a fully covariant expression for the fermion vertex operator, which is not available at present. Of course states with fermion pairs can always be obtained by factorization as above, so we can in general expect that amplitudes with external space-time fermions will also admit chiral splitting, though chiral amplitudes should now be allowed to have quadratic branch cuts between vertex operators.

\section{Conclusion}

We have investigated the analyticity properties of non-chiral correlation functions $\mathscr{A}_{\delta}$ of scalar superfields on a background supergeometry with an arbitrary fixed spin structure $\delta$ :

$$
\mathscr{A}_{\delta}=\left\langle\prod_{i=1}^{n} V\left(k_{i} ; \mathbf{z}_{i}, \overline{\mathbf{z}}_{i} ; \zeta_{i}, \bar{\zeta}_{i}\right)\right\rangle_{X} .
$$

The vertex operators we consider are the ones that describe creation and annihilation of bosonic particles in the superstring spectrum. The points $\mathbf{z}_{i}$ are insertion points on the super Riemann surface, $k_{i}^{\mu}$ are the external momenta, and $\zeta_{i}$ are the chiral polarization tensors. The main results of this paper can then be stated as

\section{Chiral Splitting Theorem}

The non-chiral correlation function $\mathscr{A}_{\delta}$ in which left and right chiralities are complex conjugates of one another and endowed with the same spin structure $\delta$, splits into a chiral amplitude $\mathscr{F}_{\delta}^{m}$ and its complex conjugate up to a local anomaly $S_{L}$

$$
\begin{aligned}
& \mathscr{A}_{\delta}\left(k_{i}^{\mu} ; \mathbf{z}_{i}, \overline{\mathbf{z}}_{i} ; \zeta_{i}, \bar{\zeta}_{i} ; \Omega_{I J}, \bar{\Omega}_{I J}, \chi_{\bar{z}}^{+}, \chi_{z}^{-}\right) \\
& \quad=(2 \pi)^{10} \delta(k) e^{10 S_{L}} \int_{\mathscr{T}} d p_{I}^{\mu}\left|\mathscr{F}_{\delta}^{m}\left(k_{i}^{\mu} ; \mathbf{z}_{i}, \zeta_{i} ; \Omega_{I J}, \chi_{\bar{z}}^{+} ; p_{I}^{\mu}\right)\right|^{2} .
\end{aligned}
$$

The chiral amplitude depends holomorphically on the background supergeometry and the chiral polarization tensors, meromorphically on the vertex operator 
insertion points. It is a form of weight $(1 / 2,0)$ in each $\mathbf{z}_{i}$, but has monodromy. The single-valuedness of the correlation function is restored upon integrating over the loop momenta $p_{I}^{\mu}$.

For vertices corresponding to massless particles in the superstring spectrum, explicit expressions in terms of contractions of effective chiral fields were given in (5.4) and (6.13), and in manifestly supersymmetric form in (5.43-45) and (6.40) for even and odd spin structures respectively. The key supergeometric notions of super abelian differentials, super period matrices, and super prime forms are introduced in (5.9), (6.25), (6.26), (5.20-22), (6.28-29), and (5.37), (6.35).

The effective rules leading to these expressions for $\mathscr{F}_{\delta}^{m}$ can now be easily formulated. One should replace the non-chiral $X=x+\theta \psi_{+}+\bar{\theta} \bar{\psi}_{-}+i \theta \bar{\theta} F$ by a chiral field $X_{+}=x_{+}+\theta \psi_{+}$, super covariant derivatives $\mathscr{D}_{+}$by their flat analogues $\partial_{+}$, drop the chiralities mixing terms in the action, and introduce $p_{I}^{\mu}$ as the momenta flowing through the loop $A_{I}$. For odd spin structure $\mathscr{F}_{\delta}^{m}$ also requires an integration over the Dirac zero mode, paired with the even abelian differential $\hat{\omega}_{0}$.

To obtain superstring scattering amplitudes from the scalar superfield correlation functions, one endows the chiral amplitudes of opposite chiralities $\mathscr{F}_{\delta}^{m}, \mathscr{F}_{\delta}^{m}$ with independent spin structures $\delta$ and $\delta$ and matches them at the same value of the internal momenta $p_{I}^{\mu}$. This gives the Type II superstrings of (7.6), (7.7), and the heterotic string of (7.8).

\section{Appendix A: Conventions}

General two-dimensional coordinates are denoted by $\xi^{1}$ and $\xi^{2}$, or $\xi=\frac{1}{\sqrt{2}}\left(\xi^{1}+i \xi^{2}\right)$. The metric is then $d s^{2}=g_{m n} d \xi^{m} d \xi^{n}$. Locally conformally flat coordinates are denoted by $z, w$, etc., and the metric becomes $d s^{2}=2 g_{z \bar{z}} d z d \bar{z}$, $d^{2} z \sqrt{g}=g_{z \bar{z}} d x d y$.

$\mathrm{U}(1)$ vector indices are denoted by $a, b, \ldots$ and take on the values $z$ and $\bar{z}$; spinor indices are denoted by $\alpha, \beta, \ldots$ and take on the values + and - . We use the same notations $z$ and $\bar{z}$ for conformally flat coordinates and $U$ (1) indices because in conformally flat coordinate systems they may be identified. Furthermore

$$
\gamma^{a} \gamma^{b}=-\frac{1}{2} \delta^{a b}-\frac{1}{2} \varepsilon^{a b} \gamma^{5}, \quad \varepsilon^{z \bar{z}}=-i
$$

We take the convenient representation of the Clifford algebra

$$
\left(\gamma^{z}\right)_{++}=\left(\gamma^{\bar{z}}\right)_{--}=1, \quad\left(\gamma_{5}\right)_{+}{ }^{+}=-\left(\gamma_{5}\right)_{-}{ }^{-}=i, \quad\left(\gamma^{a}\right)_{\alpha \beta}=0, \quad \alpha \neq \beta,
$$

and raising and lowering indices are performed as follows

$$
\psi_{+}=-\psi^{-}, \quad \psi_{-}=\psi^{+} .
$$

We use a somewhat unusual normalization of the Dirac delta function

$$
\partial_{\bar{z}} \frac{1}{z-w}=2 \pi \delta(z-w)
$$




\section{Appendix B: Contact Terms}

In this appendix we collect all contact terms between vertex insertion points that arise from the various contractions, and explain why they need not be included in the final expression for superstring scattering amplitudes.

Contractions of the auxiliary field $F^{\mu}$ give rise to the contribution $\mathscr{A}_{F}$ of (4.2), which is readily found to be

$$
\begin{aligned}
\mathscr{A}_{F}= & \exp \left[\pi \sum _ { i , j = 1 } ^ { n } \delta ( z _ { i } , z _ { j } ) \left(\theta_{i} \bar{\theta}_{i} \theta_{j} \bar{\theta}_{j} k_{i}^{\mu} k_{j}^{\mu}-2 i \zeta_{j}^{\mu} \bar{\theta}_{j} \theta_{i} \bar{\theta}_{i} k_{i}^{\mu}\right.\right. \\
& \left.\left.+2 i \bar{\zeta}_{j}^{\mu} \theta_{j} \theta_{i} \bar{\theta}_{i} k_{i}^{\mu}+\zeta_{i}^{\mu} \zeta_{j}^{\mu} \bar{\theta}_{i} \bar{\theta}_{j}+\bar{\zeta}_{i} \bar{\zeta}_{j} \theta_{i} \theta_{j}-2 \zeta_{i}^{\mu} \bar{\zeta}_{j}^{\mu} \bar{\theta}_{i} \theta_{j}\right)\right] .
\end{aligned}
$$

Contractions of the $x^{\mu}$ fields produce

$$
\exp \left[2 \pi \sum_{i, j=1}^{n} \delta\left(z_{i}, z_{j}\right)\left(\zeta_{i}^{\mu} \theta_{i}-\frac{1}{2} \bar{\zeta}_{i}^{\mu} \theta_{i} \bar{\theta}_{i} \chi_{\bar{z}}^{+}\left(z_{i}\right)\right)\left(\bar{\zeta}_{i}^{\mu} \bar{\theta}_{i}+\frac{1}{2} \zeta_{j}^{\mu} \theta_{j} \bar{\theta}_{j} \chi_{z}^{-}\left(z_{j}\right)\right)\right] .
$$

Finally the contact terms from the contractions of $\psi_{+}$are

$$
\exp \left[2 \pi \sum_{i, j=1}^{n}\left(\bar{\zeta}_{i}^{\mu} \theta_{i} \bar{\theta}_{i} \bar{\zeta}_{j}^{\mu} \theta_{j} \bar{\theta}_{j} \partial_{z_{j}} \delta\left(z_{i}, z_{j}\right)+\left(i k_{i}^{\mu} \theta_{i}+\zeta_{i}^{\mu}\right) \bar{\zeta}_{j}^{\mu} \theta_{j} \bar{\theta}_{j} \delta\left(z_{i}, z_{j}\right)+\text { c.c. }\right)\right] .
$$

The above contributions are contact terms in the sense that if the vertex operators are inserted at different points on the Riemann surface, these terms do not contribute.

Due to the presence of the $\theta$ factors, the exponentials may be expanded in a finite order power series, where any insertion point is at most common to two delta functions. Now the full amplitude always contains an overall factor of

$$
\prod_{i<j} E\left(z_{i}, z_{j}\right)^{k_{i} k_{j}} .
$$

By analytic continuation in the external momenta, all powers can always be made positive, so that the delta functions of the contact terms have no effect. Thus contact terms do not contribute, even when vertex operator positions are integrated over. This argument is equivalent to what was called the argument of the cancelled propagator in dual model theory.

\section{Appendix C: Existence of Super Holomorphic Forms}

For completeness we wish to investigate the number and nature of holomorphic forms satisfying the differential equation $\mathscr{D}_{-}^{n} V=0$. For simplicity we shall consider the case when the auxiliary field $A$ is zero. Solutions are given by

satisfying

$$
V=V_{0}^{(n)}+\theta V_{+}^{(n)}
$$

$$
\begin{aligned}
D_{\bar{z}} V_{0}^{(n)}+\frac{1}{2} \chi_{\bar{z}}^{+} V_{+}^{(n)} & =0, \\
D_{\bar{z}} V_{+}^{(n)}+\frac{1}{2} \chi_{\bar{z}}^{+} D_{z} V_{0}^{(n)}+n\left(D_{z} \chi_{\bar{z}}^{+}\right) V_{0}^{(n)} & =0 .
\end{aligned}
$$


- For $n<1 / 2$, the homogeneous equations with $\chi_{\bar{z}}^{+}=0$ have no solutions, hence $V=0$.

- For $n>1$, the adjoints of the $D_{\bar{z}}$ operators are invertible, so there are $(2 n-1)(h-1)+2 n(h-1)$ solutions, which are odd or even, depending on whether $n$ is half an odd or half an even integer. This is true for all values of $\chi_{\bar{z}}^{+}$.

- For $n=1,1 / 2,0,-1 / 2$, the preceding count of solutions still holds, but now the forms $V$ will in general have non-trivial monodromy, except for $n=1 / 2$ and even spin structure, which is the one of the cases we examined in detail in this paper.

As an example we can consider the case $n=1$, solved by

$$
V_{0}^{(1)}=\sum_{I=1}^{h} c_{I} \omega_{I}-\frac{1}{4 \pi} \int d^{2} w \partial_{z} \ln E(z, w) \chi_{\bar{w}}^{+} V_{+}^{(1)}
$$

which has monodromy through the $\partial_{z} \ln E(z, w)$ factor. On the other hand, we see that the number of solutions for the case $n=3 / 2$ is always the same independently of $\chi_{\bar{z}}^{+}$, which just corresponds to the dimension of supermoduli space.

Acknowledgements. We would like to thank A. Morozov for stimulating discussions, Ken Aoki for useful comments on the manuscript, and the Aspen Center for Physics where part of this work was carried out.

\section{References}

1. Ramond, P.: Dual theory for free fermions. Phys. Rev. D3, 2415 (1971)

Neveu, A., Schwarz, J.H.: Factorizable dual models of pions. Nucl. Phys. B31, 86 (1971)

Neveu, A., Schwarz, J.H.: Quark model of dual pions. Phys. Rev. D4, 109 (1971)

Neveu, A., Schwarz, J.H., Thorn, C.: Reformulation of the dual pion model. Phys. Lett. 35B, 525 (1971)

Thorn, C.: Embryonic dual model for pions and fermions. Phys. Rev. D4, 1112 (1971)

2. Gliozzi, F., Scherk, J., Olive, D.: Supersymmetry, supergravity theories, and the dual spinor model. Nucl. Phys. B122, 253 (1977)

Green, M. B., Schwarz, J.H.: Supersymmetrical string theory. Nucl. Phys. B18, 502 (1981)

3. Gross, D.J., Harvey, J.A., Martinec, E., Rohm, R.: Heterotic string theory (I) The free heterotic string. Nucl. Phys. B256, 253 (1986)

4. Witten, E.: Global anomalies in string theory. Argonne Conference on Anomalies, Topology and geometry, Bardeen, W., White, A., p. 61, Singapore: World Scientific 1985

5. Seiberg, N., Witten, E.: Spin structures in string theory. Nucl. Phys. B276, 272 (1986)

6. Green, M. B., Schwarz, J.H.: Supersymmetrical string theories. Phys. Lett 109 B, 444 (1982)

7. Gross, D. J., Harvey, J.A., Martinec, E., Rohm, R.: Heterotic string. Phys. Rev. Lett. 54, 502 (1985); Heterotic string (II), the interacting heterotic string. Nucl. Phys. B267, 75 (1986)

8. Gervais, J.L., Sakita, B.: Field theory interpretation of supergauges in dual models. Nucl. Phys. B34, 63 (1971)

Zumino, B.: Relativistic strings and supergauges. In: Renormalization and invariance in quantum field theory, Cainiello, E. (ed.). New York: Plenum press 1974

Brink, L., DiVecchia, P., Howe, P.: A locally symmetric and reparametrization invariant action for the spinning string. Phys. Lett. 65B, 471 (1976)

Deser, S., Zumino, B.: A complete action for the spinning string. Phys. Lett 65 B, 369 (1976)

9. Belavin, A., Knizhnik, V.G.: Phys. Lett B168, 201 (1986)

Bost, J.B., Jolicoeur, J.: Phys. Lett. B174, 279 (1986)

Catenacci, R., Cornalba, M., Martinelli, M., Reina, C.: Phys. Lett. B172, 328 (1986) 
10. D'Hoker, E., Phong, D.H.: Superholomorphic anomalies and supermoduli space. Nucl. Phys. B292, 317 (1987)

11. Polyakov, A.M.: Quantum geometry of fermionic strings. Phys. Lett. B103, 211 (1981)

12. Martinec, E.: Superspace geometry of superstrings. Phys. Rev. D28, 2604 (1983)

13. D'Hoker, E., Phong, D. H.: The geometry of string perturbation theory. Rev. Mod. Phys. 60 , 917 (1988)

14. Sonoda, H.: Berkeley Preprint LBL-24327

15. Bershadsky, M.: The loop measure over moduli space. Phys. Let. B201, 67 (1988)

16. Alvarez-Gaumé, L., Gomez, C., Nelson, P., Sierra, G., Vafa, C.: Fermionic strings in the operator formalism. Nucl. Phys. B 311, 333 (1988)

17. Belavin, A., Polyakov, A.M., Zamolodchikov, A.: Nucl. Phys. B241, 333 (1984)

18. Dotsenko, V., Fateev, V.: Nucl. Phys. B241, 312 (1984)

19. Dijkgraaf, R., Verlinde, E., Verlinde, H.: $\mathrm{c}=1$ conformal field theories. Commun. Math. Phys. 115, 649 (1988)

20. Verlinde, E., Verlinde, H.: Multiloop calculations in covariant superstring theory. Phys. Lett. B 192, 95 (1987)

21. Mumford, D.: Tata lectures on Theta I, II. Boston: Birkhäuser 1983

22. Fay, J.: Theta functions on Riemann surfaces. Lecture Notes Series vol. 352. Berlin, Heidelberg, New York: Springer 1973

23. D’Hoker, E., Phong, D.H.: Vertex operators for closed string theories. Phys. Rev. D35, 3890 (1987)

24. Howe, P.: Super-Weyl transformations in two dimensions. J. Phys. A12, 393 (1989)

25. D'Hoker, E., Phong, D.H.: Loop amplitudes for the fermionic string. Nucl Phys. B278, 225 (1986)

26. Verlinde, E., Verlinde, H.: Nucl. Phys. B288, 357 (1987)

Communicated by S.-T. Yau

Received December 15, 1988 
